\title{
Effective field theory for dark matter direct detection up to dimension seven
}

\author{
Joachim Brod, ${ }^{a, b}$ Aaron Gootjes-Dreesbach, ${ }^{a}$ Michele Tammaro ${ }^{b}$ and Jure Zupan ${ }^{b}$ \\ ${ }^{a}$ Fakultät für Physik, Technischen Universität Dortmund, \\ Dortmund D-44221, Germany \\ ${ }^{b}$ Physics Department, University of Cincinnati, \\ Cincinnati $\mathrm{OH}$ 45221, U.S.A. \\ E-mail: joachim.brod@tu-dortmund.de, \\ aaron.gootjes-dreesbach@tu-dortmund.de, tammarme@mail.uc.edu, \\ zupanje@ucmail.uc.edu
}

ABSTRACT: We present the full basis of effective operators relevant for dark matter direct detection, up to and including operators of mass dimension seven. We treat the cases where dark matter is either a Dirac fermion, a Majorana fermion, a complex scalar, or a real scalar, allowing for dark matter to furnish a general representation of the electroweak gauge group. We describe the algorithmic procedure used to obtain the minimal set of effective operators and provide the tree-level matching conditions onto the effective theory valid below the electroweak scale.

Keywords: Beyond Standard Model, Effective Field Theories

ARXIV EPRINT: 1710.10218 


\section{Contents}

1 Introduction 1

2 Low-scale interactions of fermionic DM 2

2.1 Fermionic DM coupling to quarks, gluons and photons 3

2.2 RG running 4

2.3 Couplings to nonrelativistic nucleons and nuclear response 5

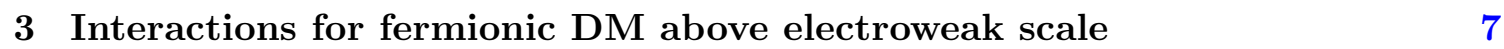

$\begin{array}{lll}3.1 & \text { EFT basis above the electroweak scale } & 7\end{array}$

3.1.1 Dimension-five operator basis $\quad 8$

3.1.2 Dimension-six operator basis $\quad 8$

3.1.3 Dimension-seven operator basis 9

$\begin{array}{lll}3.2 & \text { Tree-level matching at the electroweak scale } & 12\end{array}$

4 Scalar DM $\quad 16$

$\begin{array}{lll}\text { 4.1 Scalar DM above the electroweak scale } & 17\end{array}$

4.2 Matching below the electroweak scale, RG running, and nuclear response 18

5 Conclusions 20

$\begin{array}{ll}\text { A Construction of the operator basis } & 21\end{array}$

B Non-relativistic reduction of fermion bilinears $\quad 24$

\section{Introduction}

In order to compare results of different Dark Matter (DM) direct detection experiments one needs a theoretical framework in which to interpret the data. Since the typical momentum exchanges between DM and nuclei are small, $q_{\max } \lesssim 200 \mathrm{MeV}$, the interactions are well described within Effective Field Theory (EFT) without recourse to detailed particle physics models [1-23]. The interactions are ordered according to the dimensionality of the operators, giving an effective interaction Lagrangian

$$
\mathcal{L}_{\chi}=\sum_{a, d} \hat{\mathcal{C}}_{a}^{(d)} \mathcal{Q}_{a}^{(d)}, \quad \text { where } \quad \hat{\mathcal{C}}_{a}^{(d)}=\frac{\mathcal{C}_{a}^{(d)}}{\Lambda^{d-4}}
$$

Here, $\mathcal{C}_{a}^{(d)}$ are dimensionless Wilson coefficients, $\Lambda$ is the NP scale, generically of the order of the mediator mass, and the sum runs over the operator labels, $a$, and the mass dimension 
of the operators, $d$. The EFT description is valid as long as the mediators are heavier than the momenta exchanges, $q_{\max } \ll \Lambda$.

The UV theories of DM interactions with the SM generically give a nonzero contribution to at least one of the operators with $d \leq 7$. This set of operators already covers, for instance, all the different chiral structures one can form out of two DM and two SM fermion fields. Since the operators with low mass dimensions typically give the dominant contributions to the scattering rates, it suffices for most purposes to truncate the sum in (1.1) at $d=7$. Indeed, the commonly used DM EFTs include operators up to mass dimension seven $[24,25]$. However, the sets used in the literature are not complete - they do not contain the full basis of independent dimension-seven operators. In the present manuscript we rectify this situation.

We construct a complete EFT basis for operators up to and including dimension seven, coupling fermionic and scalar DM to the visible sector. We provide two sets of operators: a basis valid at low energies, $\mu \sim 2 \mathrm{GeV}$, as well as a basis in the unbroken electroweak phase, valid at or above the electroweak scale $\mu=\mu_{\mathrm{EW}} \sim m_{Z}$. There are a number of operators at dimension seven that were not considered before. We show that the complete basis can be chosen in such a way that these additional operators contain derivatives acting only on the DM currents. In the case of fermionic DM such operators are likely to be only a subleading correction to the operators already considered previously. The situation is qualitatively different for scalar DM. Here, the operators with derivatives acting on DM fields enter already at mass dimension six and can form the leading contribution (for instance, if DM is a pseudo-Nambu-Goldstone boson).

The formulation of the EFT at scale $\mu$ depends on which degrees of freedom are relevant at that particular scale. As a starting point we formulate in section 2.1 the EFT for fermionic DM that is valid at $\mu \sim 2 \mathrm{GeV}$ and describes the couplings of DM to the $u, d, s$ quarks, leptons, gluons, and photons. In section 2.3 we then perform a nonperturbative matching, at $\mu \sim 2 \mathrm{GeV}$, to an EFT that describes the couplings of DM to nonrelativistic protons and neutrons. Section 2.2 contains the details about the renormalization group (RG) running from the electroweak scale down to $\mu \sim 2 \mathrm{GeV}$. In section 3 we provide the necessary ingredients to relate to the EFT above the electroweak symmetry-breaking scale. In section 3.1 we first present the complete basis of dimension-seven operators above the electroweak scale, while in section 3.2 we perform the matching at the electroweak scale onto the five-flavor EFT by integrating out the Higgs, $W$, and $Z$ bosons and the top quark. In section 4 we extend the above formalism to the case of scalar DM. Section 5 contains our conclusions. Finally, in appendix A we spell out the details on the construction of the operator bases, while in appendix B we collect the relevant results for the nonrelativistic reduction of the DM and nucleon fields.

\section{Low-scale interactions of fermionic DM}

We start by considering Dirac fermion DM, with field $\chi$, and write down the complete basis of dimension-five, dimension-six, and dimension-seven operators at $\mu \sim 2 \mathrm{GeV}$, for interactions between DM and quarks, gluons, photons, and leptons, eq. (1.1). When writ- 
ing the basis we assume that there is a conserved global dark $\mathrm{U}(1)_{D}$ quantum number, which forbids currents of the form $\bar{\chi}^{c} \Gamma \chi$, where $\chi^{c}$ is the charge-conjugated DM field, and $\Gamma$ denotes a generic string of Dirac matrices. The obtained basis is valid at all scales below the electroweak scale, $\mu \lesssim M_{W}$, if matching corrections at the charm- and bottom-quark thresholds and RG running are taken into account, as detailed in section 2.2. The nonperturbative matching onto an EFT coupling DM to nonrelativistic nucleons is presented in section 2.3. We also comment on the modifications needed when considering Majorana DM.

\subsection{Fermionic DM coupling to quarks, gluons and photons}

The basis for dimension-five and dimension-six operators coincides with the one in refs. [19, 25]. The two dimension-five operators are the magnetic and electric dipole operators,

$$
\mathcal{Q}_{1}^{(5)}=\frac{e}{8 \pi^{2}}\left(\bar{\chi} \sigma^{\mu \nu} \chi\right) F_{\mu \nu}, \quad \mathcal{Q}_{2}^{(5)}=\frac{e}{8 \pi^{2}}\left(\bar{\chi} \sigma^{\mu \nu} i \gamma_{5} \chi\right) F_{\mu \nu},
$$

where $F^{\mu \nu}$ the electromagnetic field-strength tensor, and $\sigma^{\mu \nu}=\frac{i}{2}\left[\gamma^{\mu}, \gamma^{\nu}\right]$. The four dimension-six operators are formed from products of vector and axial-vector currents,

$$
\begin{array}{ll}
\mathcal{Q}_{1, f}^{(6)}=\left(\bar{\chi} \gamma^{\mu} \chi\right)\left(\bar{f} \gamma_{\mu} f\right), & \mathcal{Q}_{2, f}^{(6)}=\left(\bar{\chi} \gamma^{\mu} \gamma_{5} \chi\right)\left(\bar{f} \gamma_{\mu} f\right), \\
\mathcal{Q}_{3, f}^{(6)}=\left(\bar{\chi} \gamma^{\mu} \chi\right)\left(\bar{f} \gamma_{\mu} \gamma_{5} f\right), & \mathcal{Q}_{4, f}^{(6)}=\left(\bar{\chi} \gamma^{\mu} \gamma_{5} \chi\right)\left(\bar{f} \gamma_{\mu} \gamma_{5} f\right) .
\end{array}
$$

The subscript $f$ runs over the quark and lepton fields with masses $m_{f}<\mu$, so that $f \in$ $\left\{u, d, s, e, \mu, \tau, \nu_{e}, \nu_{\mu}, \nu_{\tau}\right\}$. (For SM neutrinos only the left-handed combination appears.) The above basis is easily extended to all scales up to the electroweak breaking scales. For scales $m_{c} \lesssim \mu \lesssim m_{b}$, in addition the charm quark is a propagating degree of freedom, so the operators with $f=c$ need to be included, and for $m_{b} \lesssim \mu \lesssim \mu_{\text {EW }}$ also the operators involving the bottom-quark field, $f=b$.

At dimension seven, there are four operators involving products of gluon field strength tensors $G^{a \mu \nu}$,

$$
\begin{array}{ll}
\mathcal{Q}_{1}^{(7)}=\frac{\alpha_{s}}{12 \pi}(\bar{\chi} \chi) G^{a \mu \nu} G_{\mu \nu}^{a}, & \mathcal{Q}_{2}^{(7)}=\frac{\alpha_{s}}{12 \pi}\left(\bar{\chi} i \gamma_{5} \chi\right) G^{a \mu \nu} G_{\mu \nu}^{a}, \\
\mathcal{Q}_{3}^{(7)}=\frac{\alpha_{s}}{8 \pi}(\bar{\chi} \chi) G^{a \mu \nu} \tilde{G}_{\mu \nu}^{a}, & \mathcal{Q}_{4}^{(7)}=\frac{\alpha_{s}}{8 \pi}\left(\bar{\chi} i \gamma_{5} \chi\right) G^{a \mu \nu} \tilde{G}_{\mu \nu}^{a},
\end{array}
$$

where $\tilde{G}_{\mu \nu}^{a}=\frac{1}{2} \varepsilon_{\mu \nu \rho \eta} G_{a}^{\rho \eta}$. There are also six operators involving a scalar or tensor fermion current. As they naturally arise from operators which, above the electroweak scale, involve a Higgs field, we include an explicit factor of $m_{f}$ and count them as dimension seven (see section 3):

$$
\begin{aligned}
\mathcal{Q}_{5, f}^{(7)} & =m_{f}(\bar{\chi} \chi)(\bar{f} f), & \mathcal{Q}_{6, f}^{(7)} & =m_{f}\left(\bar{\chi} i \gamma_{5} \chi\right)(\bar{f} f), \\
\mathcal{Q}_{7, f}^{(7)} & =m_{f}(\bar{\chi} \chi)\left(\bar{f} i \gamma_{5} f\right), & \mathcal{Q}_{8, f}^{(7)} & =m_{f}\left(\bar{\chi} i \gamma_{5} \chi\right)\left(\bar{f} i \gamma_{5} f\right), \\
\mathcal{Q}_{9, f}^{(7)} & =m_{f}\left(\bar{\chi} \sigma^{\mu \nu} \chi\right)\left(\bar{f} \sigma_{\mu \nu} f\right), & \mathcal{Q}_{10, f}^{(7)} & =m_{f}\left(\bar{\chi} \sigma^{\mu \nu} i \gamma_{5} \chi\right)\left(\bar{f} \sigma_{\mu \nu} f\right) .
\end{aligned}
$$

The operators in eqs. (2.4)-(2.8) were already used in ref. [19]. The full basis of dimensionseven operators includes, in addition, the Rayleigh operators coupling DM to two photon 
field strength tensors,

$$
\begin{aligned}
\mathcal{Q}_{11}^{(7)} & =\frac{\alpha}{12 \pi}(\bar{\chi} \chi) F^{\mu \nu} F_{\mu \nu}, & \mathcal{Q}_{12}^{(7)} & =\frac{\alpha}{12 \pi}\left(\bar{\chi} i \gamma_{5} \chi\right) F^{\mu \nu} F_{\mu \nu}, \\
\mathcal{Q}_{13}^{(7)} & =\frac{\alpha}{8 \pi}(\bar{\chi} \chi) F^{\mu \nu} \tilde{F}_{\mu \nu}, & \mathcal{Q}_{14}^{(7)} & =\frac{\alpha}{8 \pi}\left(\bar{\chi} i \gamma_{5} \chi\right) F^{\mu \nu} \tilde{F}_{\mu \nu},
\end{aligned}
$$

and eight four-fermion operators with derivatives in the DM currents,

$$
\begin{array}{llrl}
\mathcal{Q}_{15, f}^{(7)}=\left(\bar{\chi} \overleftrightarrow{i \partial}_{\mu} \chi\right)\left(\bar{f} \gamma^{\mu} f\right), & & \mathcal{Q}_{16, f}^{(7)}=\left(\bar{\chi} i \gamma_{5} \overleftrightarrow{i}_{\mu} \chi\right)\left(\bar{f} \gamma^{\mu} f\right), \\
\mathcal{Q}_{17, f}^{(7)}=\left(\bar{\chi} \overleftrightarrow{i}_{\mu} \chi\right)\left(\bar{f} \gamma^{\mu} \gamma_{5} f\right), & \mathcal{Q}_{18, f}^{(7)}=\left(\bar{\chi} i \gamma_{5} \overleftrightarrow{i} \partial_{\mu} \chi\right)\left(\bar{f} \gamma^{\mu} \gamma_{5} f\right), \\
\mathcal{Q}_{19, f}^{(7)}=\partial_{\mu}\left(\bar{\chi} \sigma^{\mu \nu} \chi\right)\left(\bar{f} \gamma_{\nu} f\right), & \mathcal{Q}_{20, f}^{(7)}=\partial_{\mu}\left(\bar{\chi} \sigma^{\mu \nu} i \gamma_{5} \chi\right)\left(\bar{f} \gamma_{\nu} f\right), \\
\mathcal{Q}_{21, f}^{(7)}=\partial_{\mu}\left(\bar{\chi} \sigma^{\mu \nu} \chi\right)\left(\bar{f} \gamma_{\nu} \gamma_{5} f\right), & \mathcal{Q}_{22, f}^{(7)}=\partial_{\mu}\left(\bar{\chi} \sigma^{\mu \nu} i \gamma_{5} \chi\right)\left(\bar{f} \gamma_{\nu} \gamma_{5} f\right),
\end{array}
$$

where $\bar{\chi} \overleftrightarrow{i}_{\mu} \chi=\bar{\chi} i \partial_{\mu} \chi-\bar{\chi} \overleftarrow{i \partial}_{\mu} \chi$, and similarly for the other bilinears.

This completes the EFT basis of dimension-seven operators for fermionic DM that couple DM bilinears to the fields in the visible sector. We have checked in two different ways that this set of operators forms a complete basis: first, using an algorithmic procedure that explicitly implements the equations of motion and various algebraic identities, outlined in appendix A, and second, with a procedure based on the conformal Hilbert series [26-28].

Note that for Majorana DM the operators $\mathcal{Q}_{1,2}^{(5)}, \quad \mathcal{Q}_{1, f}^{(6)}, \quad \mathcal{Q}_{3, f}^{(6)}, \quad \mathcal{Q}_{9, f}^{(7)}, \quad \mathcal{Q}_{10, f}^{(7)}$, and $\mathcal{Q}_{19, f}^{(7)}, \ldots, \mathcal{Q}_{22, f}^{(7)}$ vanish, while the definitions of all the other operators coventionally include an additional factor of $1 / 2$ (see also appendix A of ref. [19]).

\subsection{RG running}

If the initial conditions for Wilson coefficients are set in the four-flavor EFT, $m_{c} \lesssim \mu \lesssim m_{b}$, or in the five-flavor EFT, $m_{b} \lesssim \mu \lesssim \mu_{\mathrm{EW}}$, the Wilson coefficients $\mathcal{C}_{i}^{(d)}$ need to be evolved down to $\mu \sim 2 \mathrm{GeV}$. The RG running for operators $\mathcal{Q}_{1}^{(5)}, \ldots, \mathcal{Q}_{10, f}^{(7)}$ in eqs. (2.1)-(2.8) can be read off from ref. [8]. The effect of weak mixing below the weak scale, potentially important for dimension-six operators, eq. (2.2), was presented in ref. [29], including the leading logarithmic QCD corrections. Note that the additional dimension-seven operators with derivatives on DM fields, $\mathcal{Q}_{15, f}^{(7)}, \ldots, \mathcal{Q}_{22, f}^{(7)}$ in eqs. (2.11)-(2.14), have vanishing oneloop anomalous dimension, and there are no matching corrections at the quark thresholds.

The CP-even Rayleigh operators $\mathcal{Q}_{11}^{(7)}, \mathcal{Q}_{21}^{(7)}$ in eq. (2.9) mix into the scalar operators $\mathcal{Q}_{5, f}^{(7)}, \mathcal{Q}_{6, f}^{(7)}$ in eq. (2.6) with anomalous dimension

$$
\gamma_{11 ; 5, f}=\gamma_{12 ; 6, f}=-8\left(\frac{\alpha}{4 \pi}\right)^{2}
$$

where the running is determined by the $R G$ equations

$$
\mu \frac{d}{d \mu} C_{5, f}^{(7)}(\mu)=C_{11}^{(7)}(\mu) \gamma_{11 ; 5, f} \quad \text { and } \quad \mu \frac{d}{d \mu} C_{6, f}^{(7)}(\mu)=C_{12}^{(7)}(\mu) \gamma_{12 ; 6, f}
$$

The CP-odd Rayleigh operators $\mathcal{Q}_{13}^{(7)}, \mathcal{Q}_{14}^{(7)}$ in eq. (2.10) do not mix into the pseudo-scalar operators in eq. (2.7), contrary to their QCD counterparts, due to the absence of a QED anomaly. The RG evolution of the operators in eqs. (2.1)-(2.14) is also implemented in the DirectDM code [25]. 


\subsection{Couplings to nonrelativistic nucleons and nuclear response}

The scattering of DM on nuclei is dominated by DM scattering on single nucleons. To leading order in the chiral expansion the scattering is therefore described by the interactions of DM with nonrelativistic nucleons and protons for which the EFT Lagrangian is [5, 25],

$$
\mathcal{L}_{\mathrm{NR}}=\sum_{i, N} c_{i}^{N}\left(q^{2}\right) \mathcal{O}_{i}^{N}
$$

with two momentum-independent nonrelativistic operators,

$$
\mathcal{O}_{1}^{N}=1_{\chi} 1_{N}, \quad \mathcal{O}_{4}^{N}=\vec{S}_{\chi} \cdot \vec{S}_{N},
$$

and a set of momentum-dependent operators (displaying only the ones needed below),

$$
\begin{array}{ll}
\mathcal{O}_{5}^{N}=\vec{S}_{\chi} \cdot\left(\vec{v}_{\perp} \times \frac{i \vec{q}}{m_{N}}\right) 1_{N}, & \mathcal{O}_{6}^{N}=\left(\vec{S}_{\chi} \cdot \frac{\vec{q}}{m_{N}}\right)\left(\vec{S}_{N} \cdot \frac{\vec{q}}{m_{N}}\right), \\
\mathcal{O}_{7}^{N}=1_{\chi}\left(\vec{S}_{N} \cdot \vec{v}_{\perp}\right), & \mathcal{O}_{9}^{N}=\vec{S}_{\chi} \cdot\left(\frac{i \vec{q}}{m_{N}} \times \vec{S}_{N}\right), \\
\mathcal{O}_{11}^{N}=-\left(\vec{S}_{\chi} \cdot \frac{i \vec{q}}{m_{N}}\right) 1_{N}, & \mathcal{O}_{14}^{N}=-\left(\vec{S}_{\chi} \cdot \frac{i \vec{q}}{m_{N}}\right)\left(\vec{S}_{N} \cdot \vec{v}_{\perp}\right),
\end{array}
$$

where $N=p, n$. The momentum exchanges are defined as

$$
\vec{q}=\vec{k}_{2}-\vec{k}_{1}=\vec{p}_{1}-\vec{p}_{2}, \quad \vec{v}_{\perp}=\frac{\vec{p}_{1}+\vec{p}_{2}}{2 m_{\chi}}-\frac{\vec{k}_{1}+\vec{k}_{2}}{2 m_{N}}
$$

where $p_{1(2)}$ is the momentum of incoming (outgoing) DM particle, and similarly $k_{1(2)}$ for incoming (outgoing) nucleon.

The chirally leading nonrelativistic reduction of the operators (2.1)-(2.8) was already given in refs. $[1,19]$. The nonrelativistic reduction for the remaining dimension-seven operators with quark currents is

$$
\begin{aligned}
Q_{15, q}^{(7)} \rightarrow & 2 m_{\chi} F_{1}^{q / N} \mathcal{O}_{1}^{N}, \\
Q_{16, q}^{(7)} \rightarrow & -2 m_{N} F_{1}^{q / N} \mathcal{O}_{11}^{N}, \\
Q_{17, q}^{(7)} \rightarrow & -4 m_{\chi} F_{A}^{q / N} \mathcal{O}_{7}^{N}, \\
Q_{18, q}^{(7)} \rightarrow & 4 m_{N} F_{A}^{q / N} \mathcal{O}_{14}^{N}, \\
Q_{19, q}^{(7)} \rightarrow & \frac{\vec{q}^{2}}{2 m_{\chi}} F_{1}^{q / N} \mathcal{O}_{1}^{N}+\frac{2 \vec{q}^{2}}{m_{N}}\left(F_{1}^{q / N}+F_{2}^{q / N}\right) \mathcal{O}_{4}^{N} \\
& -2 m_{N} F_{1}^{q / N} \mathcal{O}_{5}^{N}-2 m_{N}\left(F_{1}^{q / N}+F_{2}^{q / N}\right) \mathcal{O}_{6}^{N}, \\
Q_{20, q}^{(7)} \rightarrow & -2 m_{N} F_{1}^{q / N} \mathcal{O}_{11}^{N}, \\
Q_{21, q}^{(7)} \rightarrow & -4 m_{N} F_{A}^{q / N} \mathcal{O}_{9}^{N}, \\
Q_{22, q}^{(7)} \rightarrow & 4 m_{N} F_{A}^{q / N} \mathcal{O}_{14}^{N} .
\end{aligned}
$$


The vector and axial form factors are defined through

$$
\begin{aligned}
\left\langle N^{\prime}\left|\bar{q} \gamma^{\mu} q\right| N\right\rangle & =\bar{u}_{N}^{\prime}\left[F_{1}^{q / N}\left(q^{2}\right) \gamma^{\mu}+\frac{i}{2 m_{N}} F_{2}^{q / N}\left(q^{2}\right) \sigma^{\mu \nu} q_{\nu}\right] u_{N}, \\
\left\langle N^{\prime}\left|\bar{q} \gamma^{\mu} \gamma_{5} q\right| N\right\rangle & =\bar{u}_{N}^{\prime}\left[F_{A}^{q / N}\left(q^{2}\right) \gamma^{\mu} \gamma_{5}+\frac{1}{2 m_{N}} F_{P^{\prime}}^{q / N}\left(q^{2}\right) \gamma_{5} q^{\mu}\right] u_{N},
\end{aligned}
$$

suppressing the dependence of nucleon states on their momenta, i.e., $\left\langle N^{\prime}\right| \equiv\left\langle N\left(k_{2}\right)\right|$, $|N\rangle \equiv\left|N\left(k_{1}\right)\right\rangle$, and $\bar{u}_{N}^{\prime} \equiv \bar{u}_{N}\left(k_{2}\right), u_{N} \equiv u_{N}\left(k_{1}\right)$.

The contributions of the operators $Q_{15, q}^{(7)}, \ldots, Q_{22, q}^{(7)}$ to the coefficients $c_{i}^{N}$ in the nonrelativistic Lagrangian are thus

$$
\begin{aligned}
c_{1}^{N} & =m_{\chi} F_{1}^{q / N}\left(2 \mathcal{C}_{15, q}^{(7)}+\frac{\vec{q}^{2}}{2 m_{\chi}^{2}} \mathcal{C}_{19, q}^{(7)}\right)+\cdots, \\
c_{4}^{N} & =\frac{2 \vec{q}^{2}}{m_{N}}\left(F_{1}^{q / N}+F_{2}^{q / N}\right) \mathcal{C}_{19, q}^{(7)}+\cdots, \\
c_{5}^{N} & =-2 m_{N} F_{1}^{q / N} \mathcal{C}_{19, q}^{(7)}+\cdots, \\
c_{6}^{N} & =-2 m_{N}\left(F_{1}^{q / N}+F_{2}^{q / N}\right) \mathcal{C}_{19, q}^{(7)}+\cdots, \\
c_{7}^{N} & =-4 m_{\chi} F_{A}^{q / N} \mathcal{C}_{17, q}^{(7)}+\cdots, \\
c_{9}^{N} & =-4 m_{N} F_{A}^{q / N} \mathcal{C}_{21, q}^{(7)}+\cdots \\
c_{11}^{N} & =-2 m_{N} F_{1}^{q / N}\left(\mathcal{C}_{16, q}^{(7)}+\mathcal{C}_{20, q}^{(7)}\right)+\cdots, \\
c_{14}^{N} & =4 m_{N} F_{A}^{q / N}\left(\mathcal{C}_{18, q}^{(7)}+\mathcal{C}_{22, q}^{(7)}\right) .
\end{aligned}
$$

The ellipsis denotes the contribution of the operators in eqs. (2.1)-(2.8), with the results given in eqs. (17)-(26) of ref. [25]. The coefficient $c_{14}^{N}$ receives only contributions from dimension-seven operators. In the above expressions we do not show the contributions of the Rayleigh operators, since their hadronic matrix elements are poorly known [30]. The coefficient $c_{1}^{N}$, for instance, receives a nonperturbative contribution from the CP-even Rayleigh operator, $Q_{11}^{(7)} \sim(\chi \bar{\chi}) F F$ in eq. (2.9). In the EFT with nonrelativistic nucleons one would need to keep both this contribution to $\mathcal{O}_{1}^{N}$, as well as the Rayleigh operator $Q_{11}^{(7)}$ itself, with appropriately modified Wilson coefficient due to the matching. The operators $Q_{12}^{(7)} \sim\left(\chi \bar{i} \gamma_{5} \chi\right) F F, Q_{13}^{(7)} \sim(\chi \bar{\chi}) F \tilde{F}$, and $Q_{14}^{(7)} \sim\left(\chi \bar{i} \gamma_{5} \chi\right) F \tilde{F}$ similarly give contributions in the nonperturbative matching to the non-relativistic operators $\mathcal{O}_{11}^{N} \sim \vec{S}_{\chi} \cdot \vec{q}, \mathcal{O}_{10}^{N} \sim \vec{S}_{N} \cdot \vec{q}$, and $\mathcal{O}_{6}^{N} \sim\left(\vec{S}_{\chi} \cdot \vec{q}\right)\left(\vec{S}_{N} \cdot \vec{q}\right)$, respectively. The matching also modifies the Wilson coefficients of these Rayleigh operators. At present only NDA estimates for the effects of the matching are available [30-33].

This completes the matching of the most general EFT Lagrangian for fermionic DM coupling to SM through operators up to and including dimension seven. The cross section for DM scattering on nuclei, due to DM scattering on a single nucleon, is given by eqs. (40) and (46) of ref. [5]. These expressions do receive large corrections from the 2nucleon interactions in the case of Rayleigh operators. They are of the size $\sim Z Q_{0} / m_{N}$ relative to the single-nucleon scattering, and can be dominant for large nuclei. Here $Q_{0} \sim 1 /\left(r_{0} A^{1 / 3}\right) \sim 160 \mathrm{MeV} / A^{1 / 3}$ is the momentum scale corresponding to the size of 
the nucleus, see ref. [30] for further details. For all the other UV operators the two-nucleon contributions are subleading, see, e.g., ref. [1], and the above expressions can be readily used to obtain the leading-order expressions in chiral counting for the DM-nucleus scattering cross section.

\section{Interactions for fermionic DM above electroweak scale}

If the mediators are heavier than the electroweak symmetry-breaking scale it is more convenient to write the operators in a way that is manifestly invariant under the SM gauge group. This extends the SM-EFT Lagrangian [34, 35] by adding the operators that include also DM fields.

Here, we allow DM to be part of a multiplet of the electroweak $\mathrm{SU}(2)$, with a possibly nonzero hypercharge, $Y_{\chi}$. We denote the multiplet field by $\chi$ and its electromagnetically neutral component ${ }^{1}$ (the DM) by $\chi^{0}$. We assume the DM interactions to be invariant under a global dark $\mathrm{U}(1)_{D}$ symmetry, with the $\mathrm{DM}$ multiplet charged under $\mathrm{U}(1)_{D}$.

The size of the EFT operator basis depends on the dimensionality of DM SU(2) multiplet, $d_{\chi}$. One needs to distinguish three different cases. For a DM multiplet with $d_{\chi} \geq 3$ the basis is the most general one, and is given below. If DM is a doublet, $d_{\chi}=2$, one can use the completeness relation for Pauli matrices,

$$
\sigma_{i j}^{a} \sigma_{k l}^{a}=2 \delta_{i l} \delta_{k j}-\delta_{i j} \delta_{k l}
$$

to reduce the basis to a smaller set. Below we comment on which of the operators should be dropped for the case of $d_{\chi}=2$ in order to obtain a complete set of operators without redundant members. For singlet DM, $d_{\chi}=1$, further reductions of the basis occur: because the $\mathrm{SU}(2)$ generators in that representation are zero, several operators vanish trivially.

\subsection{EFT basis above the electroweak scale}

We start the construction of EFT for DM interactions above the electroweak symmetry breaking scale by discussing the renormalizable interactions, i.e., the kinetic term in the Lagrangian

$$
\mathcal{L}=i \bar{\chi} \not D \chi-m_{\chi} \bar{\chi} \chi
$$

If $\mathrm{DM}$ is an electroweak singlet, $d_{\chi}=1, Y_{\chi}=0$, the covariant derivative is simply a partial derivative. For DM in a nontrivial representation of electroweak group the covariant derivative contains the renormalizable interactions between $\mathrm{DM}$ and the $W^{ \pm}, Z$ gauge bosons. In the important special case of vanishing hypercharge of DM, $Y_{\chi}=0$, the neutral component $\chi^{0}$ does not couple to the $Z$ boson at tree level. This suppresses the direct detection rates below the current experimental bounds.

For such cases a phenomenologically interesting possibility is that the leading interactions between DM and the visible sector, relevant for direct detection experiments, are due

\footnotetext{
${ }^{1}$ In section 2 we did not display the superscript " 0 " in order to shorten the notation.
} 
to higher dimension operators. We collect them in the effective Lagrangian

$$
\mathcal{L}_{\mathrm{EW}}=\sum_{a, d} \frac{C_{a}^{(d)}}{\Lambda^{d-4}} Q_{a}^{(d)} .
$$

Here, the label $a$ runs over different operators of dimension $d$, the $C_{a}^{(d)}$ are the corresponding dimensionless Wilson coefficients, and $\Lambda$ may be identified with the mass of the mediator.

The number of independent operators depends on whether DM is an $\mathrm{SU}(2)$ singlet, a doublet, or has $d_{\chi} \geq 3$. For general multiplet, $d_{\chi} \geq 3$, there are 8 dimension-five operators, 18 dimension-six operators, and 100 dimension-seven operators the couple DM currents to the visible sector, not counting multiplicities due to the quark and lepton flavors, and choosing a basis where all operators are Hermitian. The basis is smaller for $d_{\chi}=2\left(d_{\chi}=1\right)$, with 8 (4) dimension-five, 18 (12) dimension-six, and 92 (50) dimension-seven operators. The complete basis for dimension-five and dimension-six operators of DM interacting with the SM can be found in ref. [36], and we repeat it here for completeness. We present the complete basis of the dimension-seven operators here for the first time.

\subsubsection{Dimension-five operator basis}

The CP-conserving dimension-five operators are

$$
\begin{array}{ll}
Q_{1}^{(5)}=\frac{g_{1}}{8 \pi^{2}}\left(\bar{\chi} \sigma^{\mu \nu} \chi\right) B_{\mu \nu}, & Q_{2}^{(5)}=\frac{g_{2}}{8 \pi^{2}}\left(\bar{\chi} \sigma^{\mu \nu} \tilde{\tau}^{a} \chi\right) W_{\mu \nu}^{a}, \\
Q_{3}^{(5)}=(\bar{\chi} \chi)\left(H^{\dagger} H\right), & Q_{4}^{(5)}=\left(\bar{\chi} \tilde{\tau}^{a} \chi\right)\left(H^{\dagger} \tau^{a} H\right),
\end{array}
$$

while the CP-odd operators have an extra insertion of $\gamma_{5}$,

$$
\begin{array}{llrl}
Q_{5}^{(5)} & =\frac{g_{1}}{8 \pi^{2}}\left(\bar{\chi} \sigma^{\mu \nu} i \gamma_{5} \chi\right) B_{\mu \nu}, & & Q_{6}^{(5)}=\frac{g_{2}}{8 \pi^{2}}\left(\bar{\chi} \sigma^{\mu \nu} \tilde{\tau}^{a} i \gamma_{5} \chi\right) W_{\mu \nu}^{a}, \\
Q_{7}^{(5)}=\left(\bar{\chi} i \gamma_{5} \chi\right)\left(H^{\dagger} H\right), & Q_{8}^{(5)}=\left(\bar{\chi} \tilde{\tau}^{a} i \gamma_{5} \chi\right)\left(H^{\dagger} \tau^{a} H\right) .
\end{array}
$$

Here and below, $H$ is the SM Higgs doublet. All SU(2) (and, below, also color) indices are not displayed explicitly and are assumed to be contracted within the brackets. Note that if $\chi$ is a $\mathrm{SU}(2)$ singlet, the operators $Q_{2}^{(5)}, Q_{4}^{(5)}, Q_{6}^{(5)}$, and $Q_{8}^{(5)}$ are absent. In a perturbative UV theory the operators $Q_{1,2}^{(5)}$ and $Q_{5,6}^{(5)}$ are generated at one loop, while the operators $Q_{3,4}^{(5)}$ and $Q_{7,8}^{(5)}$ are typically generated at tree level. This expectation is reflected in our normalization of the operators.

\subsubsection{Dimension-six operator basis}

At dimension six there are many more operators. We do not consider flavor violating operators, keeping our discussion minimal. For each SM fermion generation, $i=1,2,3$, there are then eight operators that are products of DM currents and quark currents,

$$
\begin{array}{ll}
Q_{1, i}^{(6)}=\left(\bar{\chi} \gamma_{\mu} \tilde{\tau}^{a} \chi\right)\left(\bar{Q}_{L}^{i} \gamma^{\mu} \tau^{a} Q_{L}^{i}\right), & Q_{5, i}^{(6)}=\left(\bar{\chi} \gamma_{\mu} \gamma_{5} \tilde{\tau}^{a} \chi\right)\left(\bar{Q}_{L}^{i} \gamma^{\mu} \tau^{a} Q_{L}^{i}\right), \\
Q_{2, i}^{(6)}=\left(\bar{\chi} \gamma_{\mu} \chi\right)\left(\bar{Q}_{L}^{i} \gamma^{\mu} Q_{L}^{i}\right), & Q_{6, i}^{(6)}=\left(\bar{\chi} \gamma_{\mu} \gamma_{5} \chi\right)\left(\bar{Q}_{L}^{i} \gamma^{\mu} Q_{L}^{i}\right),
\end{array}
$$




$$
\begin{array}{ll}
Q_{3, i}^{(6)}=\left(\bar{\chi} \gamma_{\mu} \chi\right)\left(\bar{u}_{R}^{i} \gamma^{\mu} u_{R}^{i}\right), & Q_{7, i}^{(6)}=\left(\bar{\chi} \gamma_{\mu} \gamma_{5} \chi\right)\left(\bar{u}_{R}^{i} \gamma^{\mu} u_{R}^{i}\right), \\
Q_{4, i}^{(6)}=\left(\bar{\chi} \gamma_{\mu} \chi\right)\left(\bar{d}_{R}^{i} \gamma^{\mu} d_{R}^{i}\right), & Q_{8, i}^{(6)}=\left(\bar{\chi} \gamma_{\mu} \gamma_{5} \chi\right)\left(\bar{d}_{R}^{i} \gamma^{\mu} d_{R}^{i}\right) .
\end{array}
$$

Here $Q_{L}$ denotes the left-handed quark doublet, and $u_{R}, d_{R}$ the right-handed up- and downtype quark singlets, respectively. The corresponding operators involving lepton currents are

$$
\begin{aligned}
Q_{9, i}^{(6)} & =\left(\bar{\chi} \gamma_{\mu} \tilde{\tau}^{a} \chi\right)\left(\bar{L}_{L}^{i} \gamma^{\mu} \tau^{a} L_{L}^{i}\right), & & Q_{12, i}^{(6)}=\left(\bar{\chi} \gamma_{\mu} \gamma_{5} \tilde{\tau}^{a} \chi\right)\left(\bar{L}_{L}^{i} \gamma^{\mu} \tau^{a} L_{L}^{i}\right), \\
Q_{10, i}^{(6)} & =\left(\bar{\chi} \gamma_{\mu} \chi\right)\left(\bar{L}_{L}^{i} \gamma^{\mu} L_{L}^{i}\right), & & Q_{13, i}^{(6)}=\left(\bar{\chi} \gamma_{\mu} \gamma_{5} \chi\right)\left(\bar{L}_{L}^{i} \gamma^{\mu} L_{L}^{i}\right), \\
Q_{11, i}^{(6)} & =\left(\bar{\chi} \gamma_{\mu} \chi\right)\left(\bar{\ell}_{R}^{i} \gamma^{\mu} \ell_{R}^{i}\right), & & Q_{14, i}^{(6)}=\left(\bar{\chi} \gamma_{\mu} \gamma_{5} \chi\right)\left(\bar{\ell}_{R}^{i} \gamma^{\mu} \ell_{R}^{i}\right),
\end{aligned}
$$

where $L_{L}$ denotes the left-handed lepton doublet, and $\ell_{R}$ the right-handed down-type lepton singlet. Finally, there are four dimension-six operators involving Higgs currents,

$$
\begin{aligned}
Q_{15}^{(6)} & =\left(\bar{\chi} \gamma^{\mu} \tilde{\tau}^{a} \chi\right)\left(H^{\dagger} i \stackrel{\leftrightarrow}{D}^{a}{ }_{\mu} H\right), & & Q_{17}^{(6)}=\left(\bar{\chi} \gamma^{\mu} \gamma_{5} \tilde{\tau}^{a} \chi\right)\left(H^{\dagger} i \stackrel{\leftrightarrow}{D}^{a}{ }_{\mu} H\right), \\
Q_{16}^{(6)} & =\left(\bar{\chi} \gamma^{\mu} \chi\right)\left(H^{\dagger} i \stackrel{\leftrightarrow}{D}_{\mu} H\right), & Q_{18}^{(6)} & =\left(\bar{\chi} \gamma^{\mu} \gamma_{5} \chi\right)\left(H^{\dagger} i \stackrel{\leftrightarrow}{D}_{\mu} H\right) .
\end{aligned}
$$

The Higgs currents are defined in terms of hermitian combinations of the covariant derivatives, $\stackrel{\leftrightarrow}{D}_{\mu} \equiv D_{\mu}-\overleftarrow{\leftarrow}_{\mu}^{\dagger}$ and ${\stackrel{\leftrightarrow}{D^{a}}}_{\mu} \equiv \tau^{a} D_{\mu}-\overleftarrow{\leftarrow}_{\mu}^{\dagger} \tau^{a}$. Additional operators with covariant derivatives acting on the DM fields vanish via the DM equations of motion, up to total derivatives. As in the case of dimension-five operators, the basis simplifies if DM is a $\mathrm{SU}(2)$ singlet. In this case, the operators $Q_{1, i}^{(6)}, Q_{5, i}^{(6)}, Q_{9, i}^{(6)}, Q_{12, i}^{(6)}, Q_{15}^{(6)}$, and $Q_{17}^{(6)}$ vanish and should be dropped from the basis.

\subsubsection{Dimension-seven operator basis}

We group the dimension-seven operators into several classes, depending on how many derivatives they contain and to which SM fields they couple. Our convention for the covariant derivative is $D_{\mu} \psi_{f}=\left(\partial_{\mu}+i g_{s} T^{a} G_{\mu}^{a}-i g_{2} \tilde{\tau}^{a} W_{\mu}^{a}+i g_{1} Y_{f} B_{\mu} / 2\right) \psi_{f}$, where $T^{a}$ is the generator of $\mathrm{SU}(3)_{c}, \tilde{\tau}^{a}$ the generator of $\mathrm{SU}(2)_{L}$, both in the representation furnished by $\psi_{f}$ (for a doublet we denote it by $\tau^{a}$ ), while $Y_{f}$ is the hypercharge of $\psi_{f}$. The dual field-strength tensor is defined as $\tilde{G}_{\mu \nu}^{a}=\frac{1}{2} \varepsilon_{\mu \nu \rho \eta} G_{a}^{\rho \eta}$.

Gauge-Gauge operators. For $d_{\chi} \geq 3$ there are 22 dimension-seven operators that couple DM to two gauge field-strength tensors:

$$
\begin{aligned}
Q_{1 V}^{(7)} & =\frac{\alpha_{s}}{12 \pi}(\bar{\chi} \chi) G_{\mu \nu}^{a} G^{a, \mu \nu}, & Q_{2 V}^{(7)} & =\frac{\alpha_{s}}{12 \pi}\left(\bar{\chi} i \gamma_{5} \chi\right) G_{\mu \nu}^{a} G^{a, \mu \nu}, \\
Q_{3 V}^{(7)} & =\frac{\alpha_{s}}{8 \pi}(\bar{\chi} \chi) G_{\mu \nu}^{a} \tilde{G}^{a, \mu \nu}, & Q_{4 V}^{(7)} & =\frac{\alpha_{s}}{8 \pi}\left(\bar{\chi} i \gamma_{5} \chi\right) G_{\mu \nu}^{a} \tilde{G}^{a, \mu \nu}, \\
Q_{5 V}^{(7)} & =\frac{\alpha_{1}}{12 \pi}(\bar{\chi} \chi) B_{\mu \nu} B^{\mu \nu}, & Q_{6 V}^{(7)} & =\frac{\alpha_{1}}{12 \pi}\left(\bar{\chi} i \gamma_{5} \chi\right) B_{\mu \nu} B^{\mu \nu}, \\
Q_{7 V}^{(7)} & =\frac{\alpha_{1}}{8 \pi}(\bar{\chi} \chi) B_{\mu \nu} \tilde{B}^{\mu \nu}, & Q_{8 V}^{(7)} & =\frac{\alpha_{1}}{8 \pi}\left(\bar{\chi} i \gamma_{5} \chi\right) B_{\mu \nu} \tilde{B}^{\mu \nu}, \\
Q_{9 V}^{(7)} & =\frac{\alpha_{12}}{12 \pi}\left(\bar{\chi} \tilde{\tau}^{a} \chi\right) W_{\mu \nu}^{a} B^{\mu \nu}, & Q_{10 V}^{(7)} & =\frac{\alpha_{12}}{12 \pi}\left(\bar{\chi} i \gamma_{5} \tilde{\tau}^{a} \chi\right) W_{\mu \nu}^{a} B^{\mu \nu},
\end{aligned}
$$




$$
\begin{aligned}
Q_{11 V}^{(7)}=\frac{\alpha_{12}}{8 \pi}\left(\bar{\chi} \tilde{\tau}^{a} \chi\right) W_{\mu \nu}^{a} \tilde{B}^{\mu \nu}, & Q_{12 V}^{(7)}=\frac{\alpha_{12}}{8 \pi}\left(\bar{\chi} i \gamma_{5} \tilde{\tau}^{a} \chi\right) W_{\mu \nu}^{a} \tilde{B}^{\mu \nu}, \\
Q_{13 V}^{(7)}=\frac{\alpha_{12}}{12 \pi}\left(\bar{\chi} \sigma^{\mu \nu} \tilde{\tau}^{a} \chi\right) W_{\mu \sigma}^{a} B_{\nu}^{\sigma}, & Q_{14 V}^{(7)}=\frac{\alpha_{12}}{12 \pi}\left(\bar{\chi} \sigma^{\mu \nu} i \gamma_{5} \tilde{\tau}^{a} \chi\right) W_{\mu \sigma}^{a} B_{\nu}^{\sigma}, \\
Q_{15 V}^{(7)}=\frac{\alpha_{2}}{12 \pi}(\bar{\chi} \chi) W_{\mu \nu}^{a} W^{a, \mu \nu}, & Q_{16 V}^{(7)}=\frac{\alpha_{2}}{12 \pi}\left(\bar{\chi} i \gamma_{5} \chi\right) W_{\mu \nu}^{a} W^{a, \mu \nu}, \\
Q_{17 V}^{(7)}=\frac{\alpha_{2}}{8 \pi}(\bar{\chi} \chi) W_{\mu \nu}^{a} \tilde{W}^{a, \mu \nu}, & Q_{18 V}^{(7)}=\frac{\alpha_{2}}{8 \pi}\left(\bar{\chi} i \gamma_{5} \chi\right) W_{\mu \nu}^{a} \tilde{W}^{a, \mu \nu}, \\
Q_{19 V}^{(7)}=\frac{\alpha_{2}}{8 \pi}\left(\bar{\chi} \sigma^{\mu \nu} \tilde{\tau}^{a} \chi\right) W_{\mu \sigma}^{b} W_{\nu}^{c, \sigma} \epsilon^{a b c}, & Q_{20 V}^{(7)}=\frac{\alpha_{2}}{8 \pi}\left(\bar{\chi} \sigma^{\mu \nu} i \gamma_{5} \tilde{\tau}^{a} \chi\right) W_{\mu \sigma}^{b} W_{\nu}^{c, \sigma} \epsilon^{a b c}, \\
Q_{21 V}^{(7)}=\frac{\alpha_{2}}{12 \pi}\left(\bar{\chi} \tilde{\tau}^{\{a b\}} \chi\right) W_{\mu \nu}^{a} W^{b, \mu \nu}, & Q_{22 V}^{(7)}=\frac{\alpha_{2}}{12 \pi}\left(\bar{\chi} i \gamma_{5} \tilde{\tau}^{\{a b\}} \chi\right) W_{\mu \nu}^{a} W^{b, \mu \nu},
\end{aligned}
$$

where $\tilde{\tau}^{\{a b\}}=\tilde{\tau}^{a} \tilde{\tau}^{b}+\tilde{\tau}^{b} \tilde{\tau}^{a}$. Among those, the eleven operators $\mathcal{Q}_{k V}^{(7)}$ with $k=$ $1,4,5,8,9,12,13,15,18,19,21$ are $\mathrm{CP}$ even, while the remaining eleven are $\mathrm{CP}$ odd. In the definitions of the operators we included loop factors with appropriate gauge couplings,

$$
\alpha_{1}=\frac{g_{1}^{2}}{4 \pi}, \quad \alpha_{2}=\frac{g_{2}^{2}}{4 \pi}, \quad \alpha_{12}=\frac{g_{1} g_{2}}{4 \pi}, \quad \alpha_{s}=\frac{g_{3}^{2}}{4 \pi},
$$

since these operators arise in perturbative theories at one loop or higher.

For $d_{\chi}<3$ not all of the above operators are linearly independent. For DM that is an electroweak doublet, $d_{\chi}=2$, the operators $\mathcal{Q}_{21 V}^{(7)}, \mathcal{Q}_{22 V}^{(7)}$ should be dropped from the basis, since they are equivalent to $\mathcal{Q}_{15 V}^{(7)}, \mathcal{Q}_{16 V}^{(7)}$. Similarly, for DM that is an electroweak singlet, $d_{\chi}=1$, the operators $\mathcal{Q}_{9 V}^{(7)}, \ldots, Q_{14 V}^{(7)}$ and $\mathcal{Q}_{19 V}^{(7)}, \ldots, \mathcal{Q}_{22 V}^{(7)}$ should be dropped from the basis.

Gauge-Higgs operators. For $d_{\chi} \geq 3$ there are 12 operators that couple DM currents to a set of mixed Higgs and gauge-boson operators,

$$
\begin{array}{rlrl}
Q_{1 V^{\prime}}^{(7)} & =\frac{g_{1}}{8 \pi^{2}}\left(\bar{\chi} \sigma^{\mu \nu} \chi\right) B_{\mu \nu} H^{\dagger} H, & & Q_{2 V^{\prime}}^{(7)}=\frac{g_{1}}{8 \pi^{2}}\left(\bar{\chi} \sigma^{\mu \nu} i \gamma_{5} \chi\right) B_{\mu \nu} H^{\dagger} H, \\
Q_{3 V^{\prime}}^{(7)} & =\frac{g_{1}}{8 \pi^{2}}\left(\bar{\chi} \sigma^{\mu \nu} \tilde{\tau}^{a} \chi\right) B_{\mu \nu} H^{\dagger} \tau^{a} H, & & Q_{4 V^{\prime}}^{(7)}=\frac{g_{1}}{8 \pi^{2}}\left(\bar{\chi} \sigma^{\mu \nu} i \gamma_{5} \tilde{\tau}^{a} \chi\right) B_{\mu \nu} H^{\dagger} \tau^{a} H, \\
Q_{5 V^{\prime}}^{(7)}=\frac{g_{2}}{8 \pi^{2}}\left(\bar{\chi} \sigma^{\mu \nu} \chi\right) W_{\mu \nu}^{a} H^{\dagger} \tau^{a} H, & Q_{6 V^{\prime}}^{(7)}=\frac{g_{2}}{8 \pi^{2}}\left(\bar{\chi} \sigma^{\mu \nu} i \gamma_{5} \chi\right) W_{\mu \nu}^{a} H^{\dagger} \tau^{a} H, \\
Q_{7 V^{\prime}}^{(7)}=\frac{g_{2}}{8 \pi^{2}}\left(\bar{\chi} \sigma^{\mu \nu} \tilde{\tau}^{a} \chi\right) W_{\mu \nu}^{a} H^{\dagger} H, & Q_{8 V^{\prime}}^{(7)}=\frac{g_{2}}{8 \pi^{2}}\left(\bar{\chi} \sigma^{\mu \nu} i \gamma_{5} \tilde{\tau}^{a} \chi\right) W_{\mu \nu}^{a} H^{\dagger} H, \\
Q_{9 V^{\prime}}^{(7)}=\frac{g_{2}}{8 \pi^{2}}\left(\bar{\chi} \sigma^{\mu \nu} \tilde{\tau}^{a} \chi\right) W_{\mu \nu}^{b} H^{\dagger} \tau^{c} H \epsilon^{a b c}, & Q_{10 V^{\prime}}^{(7)}=\frac{g_{2}}{8 \pi^{2}}\left(\bar{\chi} \sigma^{\mu \nu} i \gamma_{5} \tilde{\tau}^{a} \chi\right) W_{\mu \nu}^{b} H^{\dagger} \tau^{c} H \epsilon^{a b c}, \\
Q_{11 V^{\prime}}^{(7)}=\frac{g_{2}}{8 \pi^{2}}\left(\bar{\chi} \sigma^{\mu \nu} \tilde{\tau}^{\{a b\}} \chi\right) W_{\mu \nu}^{a} H^{\dagger} \tau^{b} H, & Q_{12 V^{\prime}}^{(7)}=\frac{g_{2}}{8 \pi^{2}}\left(\bar{\chi} \sigma^{\mu \nu} i \gamma_{5} \tilde{\tau}^{\{a b\}} \chi\right) W_{\mu \nu}^{a} H^{\dagger} \tau^{b} H .
\end{array}
$$

The definitions of the operators include an appropriate gauge coupling and a loop factor, because they are expected to arise at one loop when matching from a UV theory. For DM that is part of an electroweak doublet, $d_{\chi}=2$, the operators $Q_{11 V^{\prime}}^{(7)}$ and $Q_{12 V^{\prime}}^{(7)}$ should be dropped from the basis, while for singlet DM, $d_{\chi}=1$, in addition the operators $Q_{3 V^{\prime}, 4 V^{\prime}}^{(7)}$, and $Q_{7 V^{\prime}, \ldots, 10 V^{\prime}}^{(7)}$ should be dropped. 
Four-Higgs operators. For $d_{\chi} \geq 3$ there are six dimension-seven operators that involve only DM and Higgs fields,

$$
\begin{aligned}
Q_{1 H}^{(7)}=(\bar{\chi} \chi) H^{\dagger} H H^{\dagger} H, & Q_{2 H}^{(7)}=\left(\bar{\chi} i \gamma_{5} \chi\right) H^{\dagger} H H^{\dagger} H, \\
Q_{3 H}^{(7)}=\left(\bar{\chi} \tilde{\tau}^{a} \chi\right) H^{\dagger} \tau^{a} H H^{\dagger} H, & Q_{4 H}^{(7)}=\left(\bar{\chi} i \gamma_{5} \tilde{\tau}^{a} \chi\right) H^{\dagger} \tau^{a} H H^{\dagger} H, \\
Q_{5 H}^{(7)}=\left(\bar{\chi} \tilde{\tau}^{\{a b\}} \chi\right)\left(H^{\dagger} \tau^{a} H\right)\left(H^{\dagger} \tau^{b} H\right), & Q_{6 H}^{(7)}=\left(\bar{\chi} i \gamma_{5} \tilde{\tau}^{\{a b\}} \tilde{\chi}\right)\left(H^{\dagger} \tau^{a} H\right)\left(H^{\dagger} \tau^{b} H\right) .
\end{aligned}
$$

The operators $Q_{1 H}^{(7)}, Q_{3 H}^{(7)}$, and $Q_{5 H}^{(7)}$ are CP even, while $Q_{2 H}^{(7)}, Q_{4 H}^{(7)}$, and $Q_{6 H}^{(7)}$ are CP odd. For DM that is an electroweak singlet, $d_{\chi}=1$, the operators $Q_{3 H}^{(7)}, \ldots, Q_{6 H}^{(7)}$ are redundant and should be dropped, while for DM that is a doublet, $d_{\chi}=2$, only the operators $Q_{5 H}^{(7)}$ and $Q_{6 H}^{(7)}$ should be dropped from the basis.

Two-Higgs operators. There are ten operators coupling a $d_{\chi} \geq 3 \mathrm{DM}$ multiplet to Higgs bilinears, given by

$$
\begin{array}{lll}
Q_{1 H^{\prime}}^{(7)}=(\bar{\chi} \chi) D_{\mu} H^{\dagger} D^{\mu} H, & Q_{2 H^{\prime}}^{(7)}=\left(\bar{\chi} i \gamma_{5} \chi\right) D_{\mu} H^{\dagger} D^{\mu} H \\
Q_{3 H^{\prime}}^{(7)}=\left(\bar{\chi} \tilde{\tau}^{a} \chi\right) D_{\mu} H^{\dagger} \tau^{a} D^{\mu} H, & Q_{4 H^{\prime}}^{(7)}=\left(\bar{\chi} i \gamma_{5} \tilde{\tau}^{a} \chi\right) D_{\mu} H^{\dagger} \tau^{a} D^{\mu} H \\
Q_{5 H^{\prime}}^{(7)}=i\left(\bar{\chi} \sigma^{\mu \nu} \chi\right) D_{\mu} H^{\dagger} D_{\nu} H, & Q_{6 H^{\prime}}^{(7)}=i\left(\bar{\chi} \sigma^{\mu \nu} i \gamma_{5} \chi\right) D_{\mu} H^{\dagger} D_{\nu} H \\
Q_{7 H^{\prime}}^{(7)}=i\left(\bar{\chi} \sigma^{\mu \nu} \tilde{\tau}^{a} \chi\right) D_{\mu} H^{\dagger} \tau^{a} D_{\nu} H, & Q_{8 H^{\prime}}^{(7)}=i\left(\bar{\chi} \sigma^{\mu \nu} i \gamma_{5} \tilde{\tau}^{a} \chi\right) D_{\mu} H^{\dagger} \tau^{a} D_{\nu} H . \\
Q_{9 H^{\prime}}^{(7)}=i\left(\bar{\chi} \sigma^{\mu \nu} \tilde{\tau}^{\{a b\}} \chi\right)\left(D_{\mu} \tau^{a} H^{\dagger}\right)\left(D_{\nu} \tau^{b} H\right), & Q_{10 H^{\prime}}^{(7)}=i\left(\bar{\chi} \sigma^{\mu \nu} \tilde{\tau}^{\{a b\}} i \gamma_{5} \chi\right)\left(D_{\mu} \tau^{a} H^{\dagger}\right)\left(D_{\nu} \tau^{b} H\right) .
\end{array}
$$

For DM that is an electroweak doublet, $d_{\chi}=2$, the operators $Q_{9 H^{\prime}}^{(7)}$ and $Q_{10 H^{\prime}}^{(7)}$ are redundant and should be dropped from the basis. Similarly, for electroweak singlet DM, $d_{\chi}=1$, the operators $Q_{3 H^{\prime}}^{(7)}, Q_{4 H^{\prime}}^{(7)}, Q_{7 H^{\prime}, \ldots, 10 H^{\prime}}^{(7)}$ should be dropped.

Yukawa-like operators. The operators with chirality-flipping currents on both the DM and the SM side are

$$
\begin{array}{rlrl}
Q_{1 Y, i}^{(7)} & =(\bar{\chi} \chi)\left(\bar{Q}_{L}^{i} u_{R}^{i} \tilde{H}\right), & & Q_{2 Y, i}^{(7)}=\left(\bar{\chi} i \gamma_{5} \chi\right)\left(\bar{Q}_{L}^{i} u_{R}^{i} \tilde{H}\right), \\
Q_{3 Y, i}^{(7)}=\left(\bar{\chi} \tilde{\tau}^{a} \chi\right)\left(\bar{Q}_{L}^{i} u_{R}^{i} \tau^{a} \tilde{H}\right), & & Q_{4 Y, i}^{(7)}=\left(\bar{\chi} i \gamma_{5} \tilde{\tau}^{a} \chi\right)\left(\bar{Q}_{L}^{i} u_{R}^{i} \tau^{a} \tilde{H}\right), \\
Q_{5 Y, i}^{(7)} & =\left(\bar{\chi} \sigma_{\mu \nu} \chi\right)\left(\bar{Q}_{L}^{i} \sigma^{\mu \nu} u_{R}^{i} \tilde{H}\right), & & Q_{6 Y, i}^{(7)}=\left(\bar{\chi} \sigma_{\mu \nu} \tilde{\tau}^{a} \chi\right)\left(\bar{Q}_{L}^{i} \sigma^{\mu \nu} \tau^{a} u_{R}^{i} \tilde{H}\right), \\
Q_{7 Y, i}^{(7)} & =(\bar{\chi} \chi)\left(\bar{Q}_{L}^{i} d_{R}^{i} H\right), & & Q_{8 Y, i}^{(7)}=\left(\bar{\chi} i \gamma_{5} \chi\right)\left(\bar{Q}_{L}^{i} d_{R}^{i} H\right), \\
Q_{9 Y, i}^{(7)} & =\left(\bar{\chi} \tilde{\tau}^{a} \chi\right)\left(\bar{Q}_{L}^{i} d_{R}^{i} \tau^{a} H\right), & & Q_{10 Y, i}^{(7)}=\left(\bar{\chi} i \gamma_{5} \tilde{\tau}^{a} \chi\right)\left(\bar{Q}_{L}^{i} d_{R}^{i} \tau^{a} H\right), \\
Q_{11 Y, i}^{(7)}=\left(\bar{\chi} \sigma_{\mu \nu} \chi\right)\left(\bar{Q}_{L}^{i} \sigma^{\mu \nu} d_{R}^{i} H\right), & Q_{12 Y, i}^{(7)}=\left(\bar{\chi} \sigma_{\mu \nu} \tilde{\tau}^{a} \chi\right)\left(\bar{Q}_{L}^{i} \sigma^{\mu \nu} \tau^{a} d_{R}^{i} H\right), \\
Q_{13 Y, i}^{(7)}=(\bar{\chi} \chi)\left(\bar{L}_{L}^{i} \ell_{R}^{i} H\right), & Q_{14 Y, i}^{(7)}=\left(\bar{\chi} i \gamma_{5} \chi\right)\left(\bar{L}_{L}^{i} \ell_{R}^{i} H\right), \\
Q_{15 Y, i}^{(7)}=\left(\bar{\chi} \tilde{\tau}^{a} \chi\right)\left(\bar{L}_{L}^{i} \ell_{R}^{i} \tau^{a} H\right), & Q_{16 Y, i}^{(7)}=\left(\bar{\chi} i \gamma_{5} \tilde{\tau}^{a} \chi\right)\left(\bar{L}_{L}^{i} \ell_{R}^{i} \tau^{a} H\right), \\
Q_{17 Y, i}^{(7)}=\left(\bar{\chi} \sigma_{\mu \nu} \chi\right)\left(\bar{L}_{L}^{i} \sigma^{\mu \nu} \ell_{R}^{i} H\right), & & Q_{18 Y, i}^{(7)}=\left(\bar{\chi} \sigma_{\mu \nu} \tilde{\tau}^{a} \chi\right)\left(\bar{L}_{L}^{i} \sigma^{\mu \nu} \ell_{R}^{i} \tau^{a} H\right),
\end{array}
$$


where $\tilde{H}=i \sigma_{2} H^{*}$ is the charge-conjugated Higgs field. The above operators are not Hermitian so that they enter the effective Lagrangian, eq. (3.3), with complex coefficients. Their Hermitian conjugates have to be included, modifying (3.3) to

$$
\mathcal{L}_{\mathrm{EW}}=\sum_{a, d} \frac{C_{a}^{(d)}}{\Lambda^{d-4}} Q_{a}^{(d)}(+ \text { h.c. }),
$$

where the "h.c." is present in the sum only for the operators $Q_{1 Y, i}^{(7)}, \ldots, Q_{18 Y, i}^{(7)}$. Alternatively, one could work with a set of 36 Hermitian operators with real coefficients, by defining $Q_{i+}^{(7)}=Q_{i}^{(7)}+\left(Q_{i}^{(7)}\right)^{\dagger}, Q_{i-}^{(7)}=i Q_{i}^{(7)}-i\left(Q_{i}^{(7)}\right)^{\dagger}$, for each of the operators in eqs. (3.43)-(3.51). The above set of operators $Q_{k Y, i}^{(7)}$ is part of a complete basis for both $d_{\chi} \geq 3$ and for $d_{\chi}=2$, while if DM is an electroweak singlet, $d_{\chi}=1$, the operators with $k=3,4,6,9,10,12,15,16,18$, should be dropped from the basis.

Four-Fermion operators. Finally, we list the tensor operators that involve four fermion fields and one derivative, which for $d_{\chi} \geq 3$ and $d_{\chi}=2$ are given by

$$
\begin{array}{rlrl}
Q_{1 F, i}^{(7)} & =\partial_{\mu}\left(\bar{\chi} \sigma^{\mu \nu} \tilde{\tau}^{a} \chi\right)\left(\bar{Q}_{L}^{i} \tau^{a} \gamma_{\nu} Q_{L}^{i}\right), & & Q_{2 F, i}^{(7)}=\partial_{\mu}\left(\bar{\chi} \sigma^{\mu \nu} i \gamma_{5} \tilde{\tau}^{a} \chi\right)\left(\bar{Q}_{L}^{i} \tau^{a} \gamma_{\nu} Q_{L}^{i}\right), \\
Q_{3 F, i}^{(7)}=\partial_{\mu}\left(\bar{\chi} \sigma^{\mu \nu} \chi\right)\left(\bar{Q}_{L}^{i} \gamma_{\nu} Q_{L}^{i}\right), & & Q_{4 F, i}^{(7)}=\partial_{\mu}\left(\bar{\chi} \sigma^{\mu \nu} i \gamma_{5} \chi\right)\left(\bar{Q}_{L}^{i} \gamma_{\nu} Q_{L}^{i}\right), \\
Q_{5 F, i}^{(7)}=\partial_{\mu}\left(\bar{\chi} \sigma^{\mu \nu} \chi\right)\left(\bar{u}_{R}^{i} \gamma_{\nu} u_{R}^{i}\right), & & Q_{6 F, i}^{(7)}=\partial_{\mu}\left(\bar{\chi} \sigma^{\mu \nu} i \gamma_{5} \chi\right)\left(\bar{u}_{R}^{i} \gamma_{\nu} u_{R}^{i}\right), \\
Q_{7 F, i}^{(7)}=\partial_{\mu}\left(\bar{\chi} \sigma^{\mu \nu} \chi\right)\left(\bar{d}_{R}^{i} \gamma_{\nu} d_{R}^{i}\right), & & Q_{8 F, i}^{(7)}=\partial_{\mu}\left(\bar{\chi} \sigma^{\mu \nu} i \gamma_{5} \chi\right)\left(\bar{d}_{R}^{i} \gamma_{\nu} d_{R}^{i}\right), \\
Q_{9 F, i}^{(7)}=\partial_{\mu}\left(\bar{\chi} \sigma^{\mu \nu} \tilde{\tau}^{a} \chi\right)\left(\bar{L}_{L}^{i} \tau^{a} \gamma_{\nu} L_{L}^{i}\right), & & Q_{10 F, i}^{(7)}=\partial_{\mu}\left(\bar{\chi} \sigma^{\mu \nu} i \gamma_{5} \tilde{\tau}^{a} \chi\right)\left(\bar{L}_{L}^{i} \tau^{a} \gamma_{\nu} L_{L}^{i}\right), \\
Q_{11 F, i}^{(7)}=\partial_{\mu}\left(\bar{\chi} \sigma^{\mu \nu} \chi\right)\left(\bar{L}_{L}^{i} \gamma_{\nu} L_{L}^{i}\right), & & Q_{12 F, i}^{(7)}=\partial_{\mu}\left(\bar{\chi} \sigma^{\mu \nu} i \gamma_{5} \chi\right)\left(\bar{L}_{L}^{i} \gamma_{\nu} L_{L}^{i}\right), \\
Q_{13 F, i}^{(7)}=\partial_{\mu}\left(\bar{\chi} \sigma^{\mu \nu} \chi\right)\left(\bar{\ell}_{R}^{i} \gamma_{\nu} \ell_{R}^{i}\right), & & Q_{14 F, i}^{(7)}=\partial_{\mu}\left(\bar{\chi} \sigma^{\mu \nu} i \gamma_{5} \chi\right)\left(\bar{\ell}_{R}^{i} \gamma_{\nu} \ell_{R}^{i}\right) .
\end{array}
$$

The operators $Q_{1 F, i}^{(7)}, Q_{2 F, i}^{(7)}$ and $Q_{9 F, i}^{(7)}, Q_{10 F, i}^{(7)}$ should be dropped from the basis for singlet $\mathrm{DM}, d_{\chi}=1$.

This completes the list of dimension-seven operators coupling Dirac-fermion DM currents to the visible sector. If DM is a Majorana particle, several of these operators vanish identically. These are the operators that involve a DM vector or tensor current, namely the gauge-gauge operators $Q_{13 V}^{(7)}, Q_{14 V}^{(7)}, Q_{19 V}^{(7)}, Q_{20 V}^{(7)}$, in eqs. (3.23), (3.26); all of the gauge-Higgs operators $Q_{1 V^{\prime}}^{(7)}, \ldots, Q_{12 V^{\prime}}^{(7)}$, in eqs. (3.29)-(3.34), the two-Higgs operators $Q_{5 H^{\prime}}^{(7)}, \ldots, Q_{10 H^{\prime}}^{(7)}$, in eqs. (3.40)-(3.42); the Yukawa-like operators $Q_{5 Y, i}^{(7)}, Q_{6 Y, i}^{(7)}, Q_{11 Y, i}^{(7)}, Q_{12 Y, i}^{(7)}, Q_{17 Y, i}^{(7)}, Q_{18 Y, i}^{(7)}$ in eqs. (3.45), (3.48), and (3.51); and all of the four-fermion operators $Q_{1 F, i}^{(7)}, \ldots, Q_{14 F, i}^{(7)}$ in eqs. (3.53)-(3.59).

\subsection{Tree-level matching at the electroweak scale}

Next, we perform the tree-level matching of the EFT for DM interactions above the electroweak scale onto the EFT valid below the electroweak scale. In the matching at $\mu \sim \mu_{E W}$ we integrate out the heavy states with masses of the order of the electroweak scale - the 
Higgs boson, the top quark, and the $W$ and $Z$ bosons — resulting in the effective Lagrangian (1.1) with five active quark flavors. The results for the gauge-invariant EFT containing dimension-five and dimension-six operators can be found in ref. [36]. Here, we present the additional contributions due to the presence of dimension-seven operators.

We use the conventions of ref. [37]. The gauge fields in the broken phase are

$$
W_{\mu}^{ \pm}=\frac{1}{2}\left(W_{\mu}^{1} \mp i W_{\mu}^{2}\right), \quad\left(\begin{array}{c}
Z_{\mu} \\
A_{\mu}
\end{array}\right)=\left(\begin{array}{cc}
c_{w} & s_{w} \\
-s_{w} & c_{w}
\end{array}\right)\left(\begin{array}{c}
W_{\mu}^{3} \\
B_{\mu}
\end{array}\right),
$$

with the sine of the weak mixing angle $s_{w} \equiv g_{1} / \sqrt{g_{1}^{2}+g_{2}^{2}}$. Accordingly, we choose the following explicit form of the $\mathrm{SU}(2)$ generators

$$
\left(\tilde{\tau}^{1} \pm i \tilde{\tau}^{2}\right)_{k l}=\delta_{k, l \pm 1} \sqrt{\left(I_{\chi} \mp l\right)\left(I_{\chi} \pm l+1\right)}, \quad\left(\tilde{\tau}^{3}\right)_{k l}=l \delta_{k, l}
$$

with $I_{\chi}=\left(d_{\chi}-1\right) / 2$, and the indices $k, l$ running over the values $-I_{\chi}, \ldots, I_{\chi}-1, I_{\chi}$. The Higgs doublet field is

$$
H=\left(\begin{array}{c}
G^{+} \\
\frac{1}{\sqrt{2}}\left(v+h+i G^{0}\right)
\end{array}\right)
$$

where $h$ denotes the physical Higgs field, and $G^{ \pm}, G^{0}$ the pseudo-Goldstone fields, eaten by the longitudinal components of $W^{ \pm}$and $Z$.

Before proceeding we remark that both the DM mass, $m_{\chi}$, and the DM field $\chi$ get shifted by the contributions of the four-Higgs operators in eqs. (3.35)-(3.37) if all Higgs fields are replaced by their vacuum expaectation values. The kinetic and mass terms in the dimension-four Lagrangian eq. (3.2) thus become

$$
\left.\mathcal{L}_{\chi}^{(4)}\right|_{n_{f}=5}=i \bar{\chi}^{\prime} \not \partial \chi^{\prime}-m_{\chi}^{\prime} \bar{\chi}^{\prime} \chi^{\prime}
$$

where the $\chi$ field now only denotes the DM field, i.e., the neutral component of the DM multiplet, which in addition has been transformed by a simple chiral rotation, $\chi^{\prime}=\exp \left(\frac{i}{2} \gamma_{5} \phi\right) \chi$, where (see also ref. [38])

$$
\tan \phi=\frac{C_{7}^{(5)}+\frac{Y_{\chi}}{4} C_{8}^{(5)}+C_{2 H}^{(7)}+\frac{Y_{\chi}}{4} C_{4 H}^{(7)}+\frac{Y_{\chi}}{8} C_{6 H}^{(7)}}{C_{3}^{(5)}+\frac{Y_{\chi}}{4} C_{4}^{(5)}-2 m_{\chi} \Lambda / v_{\mathrm{EW}}^{2}+C_{1 H}^{(7)}+\frac{Y_{\chi}}{4} C_{3 H}^{(7)}+\frac{Y_{\chi}}{8} C_{5 H}^{(7)}-\frac{4 m_{\chi} \Lambda^{3}}{v^{4}}},
$$

while the new mass term is

$$
\begin{gathered}
m_{\chi}^{\prime}=m_{\chi} \cos \phi+\frac{v^{2}}{2 \Lambda}\left[\left(C_{7}^{(5)}+\frac{Y_{\chi}}{4} C_{8}^{(5)}\right) \sin \phi-\left(C_{3}^{(5)}+\frac{Y_{\chi}}{4} C_{4}^{(5)}\right) \cos \phi\right] \\
-\frac{v^{4}}{4 \Lambda^{3}}\left[\left(C_{2 H}^{(7)}+\frac{Y_{\chi}}{4} C_{4 H}^{(7)}+\frac{Y_{\chi}}{8} C_{6 H}^{(7)}\right) \sin \phi\right. \\
\left.+\left(C_{1 H}^{(7)}+\frac{Y_{\chi}}{4} C_{3 H}^{(7)}+\frac{Y_{\chi}}{8} C_{5 H}^{(7)}\right) \cos \phi\right] .
\end{gathered}
$$

The corrections from dimension-five and dimension-six operators were already derived in ref. [36]. 
The field redefinition also changes the operators $Q_{1}^{(5)}, \ldots, Q_{8}^{(5)}$ in (3.4)-(3.7) and the corresponding Wilson coefficients, $C_{i}^{(5) \prime}=C_{i}^{(5)} \cos \phi+C_{i+4}^{(5)} \sin \phi, C_{i+4}^{(5) \prime}=C_{i+4}^{(5)} \cos \phi-$ $C_{i}^{(5)} \sin \phi$, for $i=1, \ldots, 4$, while there is no change in the dimension-six Wilson coefficients. The field redefinition changes all of the dimension-seven operators in this section. The corresponding Wilson coefficients of the operators with primed fields are given by

$$
C_{2 k-1, A(2 k, A)}^{(7) \prime}=C_{2 k-1, A(2 k, A)}^{(7)} \cos \phi \pm C_{2 k, A(2 k-1, A)}^{(7)} \sin \phi,
$$

for $A=V\left(V^{\prime}, H, H^{\prime}, F\right)$, in which case $k=1, \ldots, 11(6,3,5,7)$, and also for $A=Y$, with $k=1,2,4,5,7,8$, while for the remaining dimension-seven Yukawa-like operators one has

$$
C_{k, Y}^{(7) \prime}=C_{k, Y}^{(7)} \exp (i \phi), \quad k=5,6,11,12,17,18 .
$$

From now on we will assume that the above field and mass redefinitions have been performed, and drop the primes on the Wilson coefficients, the $\chi$ fields, and the DM mass $m_{\chi}$.

Next we give the contributions from dimension-seven operators above the electroweak scale, when matching onto the EFT below the electroweak scale (the contributions from dimension-five and dimension-six operators are given in [36]). The gauge-Higgs operators, eqs. (3.29)-(3.33), contribute to the dimension-five dipole operators after the Higgs obtains its vacuum expectation value, leading to

$$
\hat{\mathcal{C}}_{1(2)}^{(5)}=\frac{v_{\mathrm{EW}}^{2}}{2 \Lambda^{3}}\left[C_{1(2) V^{\prime}}^{(7)}+\frac{Y_{\chi}}{4} C_{3(4) V^{\prime}}^{(7)}+\frac{1}{2} C_{5(6) V^{\prime}}^{(7)}+\frac{Y_{\chi}}{2} C_{7(8) V^{\prime}}^{(7)}\right]+\ldots
$$

The ellipsis denotes the tree-level contributions from the dimension-five UV operators, as well as the one-loop contributions from renormalizable gauge interactions. The explicit expressions for both of these contributions can be found in ref. [36]. Note that there are no tree-level contributions from the operators $Q_{9 V^{\prime}}^{(7)}$ and $Q_{10 V^{\prime}}^{(7)}$.

The matching of dimension-seven electroweak operators (3.17), (3.18) onto the gluonic operators $(2.4),(2.5)$ is given by

$$
\hat{\mathcal{C}}_{k}^{(7)}=\frac{1}{\Lambda^{3}} \cdot C_{(k) V}^{(7)}+\ldots, \quad k=1, \ldots, 4 .
$$

The ellipsis denotes the one-loop contribution of the dimension-five scalar operators, given in ref. [36].

The tree-level matching of the operators (3.19)-(3.27) onto the Rayleigh operators $(2.9)-(2.10)$ is given by

$$
\hat{\mathcal{C}}_{11+k}^{(7)}=\frac{1}{\Lambda^{3}}\left[C_{(5+k) V}^{(7)}+\frac{Y_{\chi}}{2} C_{(9+k) V}^{(7)}+C_{(15+k) V}^{(7)}+\left(\delta_{k 0}+\delta_{k 1}\right) \frac{Y_{\chi}^{2}}{2} C_{(21+k) V}^{(7)}\right], \quad k=0, \ldots, 3 .
$$

Note that there are no tree-level contributions to the interactions of the neutral component $\chi^{0}$ from the operators $Q_{13 V}^{(7)}, Q_{14 V}^{(7)}, Q_{19 V}^{(7)}$, and $Q_{20 V}^{(7)}$.

The Yukawa-like operators, eq. (3.43)-(3.51), match onto the dimension-seven scalarcurrent four-fermion operators, (2.6)-(2.8), by replacing the Higgs with its vacuum expectation value. In addition, the four-Higgs operators in eqs. (3.35)-(3.37) contribute to 


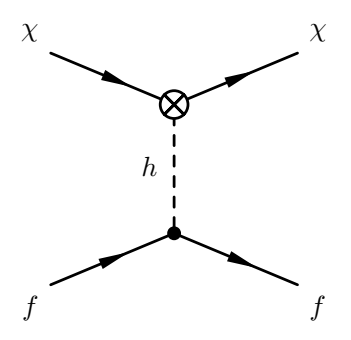

Figure 1. Matching contributions to dimension-seven effective operators for $\mu<v_{\mathrm{EW}}$ due to Higgs exchange.

the same operators via tree-level Higgs exchange, see figure 1. This yields the following coefficients for up-type quarks $\left(u_{1}=u, u_{2}=c\right)$

$$
\begin{aligned}
\hat{\mathcal{C}}_{5(6), u_{i}}^{(7)}= & \frac{1}{\Lambda^{3}}\left[\frac{v_{\mathrm{EW}}}{\sqrt{2} m_{u_{i}}} \operatorname{Re}\left(C_{1(2) Y, i}^{(7)}-\frac{Y_{\chi}}{4} C_{3(4) Y, i}^{(7)}\right)\right. \\
& \left.\quad-\frac{v_{\mathrm{EW}}^{2}}{M_{h}^{2}}\left(C_{1(2) H}^{(7)}+\frac{Y_{\chi}}{4} C_{3(4) H}^{(7)}+\frac{Y_{\chi}^{2}}{8} C_{5(6) H}^{(7)}\right)\right]+\cdots, \\
\hat{\mathcal{C}}_{7(8), u_{i}}^{(7)}= & \frac{1}{\Lambda^{3}} \frac{v_{\mathrm{EW}}}{\sqrt{2} m_{u_{i}}} \operatorname{Im}\left(C_{1(2) Y, i}^{(7)}-\frac{Y_{\chi}}{4} C_{3(4) Y, i}^{(7)}\right) \\
\hat{\mathcal{C}}_{9, u_{i}}^{(7)}= & \frac{1}{\Lambda^{3}} \frac{v_{\mathrm{EW}}}{\sqrt{2} m_{u_{i}}} \operatorname{Re}\left(C_{5 Y, i}^{(7)}-\frac{Y_{\chi}}{4} C_{6 Y, i}^{(7)}\right) \\
\hat{\mathcal{C}}_{10, u_{i}}^{(7)}= & \frac{1}{\Lambda^{3}} \frac{v_{\mathrm{EW}}}{\sqrt{2} m_{u_{i}}} \operatorname{Im}\left(C_{5 Y, i}^{(7)}-\frac{Y_{\chi}}{4} C_{6 Y, i}^{(7)}\right)
\end{aligned}
$$

where the ellipsis in eq. (3.71) denotes the contributions from dimension-five operators, given in ref. [36]. For down-type quarks $\left(d_{1}=d, d_{2}=s, d_{3}=b\right)$ one has

$$
\begin{aligned}
\hat{\mathcal{C}}_{5(6), d_{i}}^{(7)}=\frac{1}{\Lambda^{3}}\left[\frac{v_{\mathrm{EW}}}{\sqrt{2} m_{d_{i}}} \operatorname{Re}\left(C_{7(8) Y, i}^{(7)}+\frac{Y_{\chi}}{4} C_{9(10) Y, i}^{(7)}\right)\right. & \left.-\frac{v_{\mathrm{EW}}^{2}}{M_{h}^{2}}\left(C_{1(2) H}^{(7)}+\frac{Y_{\chi}}{4} C_{3(4) H}^{(7)}+\frac{Y_{\chi}^{2}}{8} C_{5(6) H}^{(7)}\right)\right]+\cdots, \\
\hat{\mathcal{C}}_{7(8), d_{i}}^{(7)}= & \frac{1}{\Lambda^{3}} \frac{v_{\mathrm{EW}}}{\sqrt{2} m_{d_{i}}} \operatorname{Im}\left(C_{7(8) Y, i}^{(7)}+\frac{Y_{\chi}}{4} C_{9(10) Y, i}^{(7)}\right), \\
\hat{\mathcal{C}}_{9, d_{i}}^{(7)}= & \frac{1}{\Lambda^{3}} \frac{v_{\mathrm{EW}}}{\sqrt{2} m_{d_{i}}} \operatorname{Re}\left(C_{11 Y, i}^{(7)}+\frac{Y_{\chi}}{4} C_{12 Y, i}^{(7)}\right), \\
\hat{\mathcal{C}}_{10, d_{i}}^{(7)}= & \frac{1}{\Lambda^{3}} \frac{v_{\mathrm{EW}}}{\sqrt{2} m_{d_{i}}} \operatorname{Im}\left(C_{11 Y, i}^{(7)}+\frac{Y_{\chi}}{4} C_{12 Y, i}^{(7)}\right),
\end{aligned}
$$

while for charged leptons $\left(\ell_{1}=e, \ell_{2}=\mu, \ell_{3}=\tau\right)$

$$
\begin{aligned}
\hat{\mathcal{C}}_{5(6), \ell_{i}}^{(7)}=\frac{1}{\Lambda^{3}}\left[\frac{v_{\mathrm{EW}}}{\sqrt{2} m_{\ell_{i}}} \operatorname{Re}\left(C_{13(14) Y, i}^{(7)}+\frac{Y_{\chi}}{4} C_{15(16) Y, i}^{(7)}\right)\right. \\
\left.\quad-\frac{v_{\mathrm{EW}}^{2}}{M_{h}^{2}}\left(C_{1(2) H}^{(7)}+\frac{Y_{\chi}}{4} C_{3(4) H}^{(7)}+\frac{Y_{\chi}^{2}}{8} C_{5(6) H}^{(7)}\right)\right]+\cdots,
\end{aligned}
$$




$$
\begin{aligned}
\hat{\mathcal{C}}_{7(8), \ell_{i}}^{(7)} & =\frac{1}{\Lambda^{3}} \frac{v_{\mathrm{EW}}}{\sqrt{2} m_{\ell_{i}}} \operatorname{Im}\left(C_{13(14) Y, i}^{(7)}+\frac{Y_{\chi}}{4} C_{15(16) Y, i}^{(7)}\right), \\
\hat{\mathcal{C}}_{9, \ell_{i}}^{(7)} & =\frac{1}{\Lambda^{3}} \frac{v_{\mathrm{EW}}}{\sqrt{2} m_{\ell_{i}}} \operatorname{Re}\left(C_{17 Y, i}^{(7)}+\frac{Y_{\chi}}{4} C_{18 Y, i}^{(7)}\right) \\
\hat{\mathcal{C}}_{10, \ell_{i}}^{(7)} & =\frac{1}{\Lambda^{3}} \frac{v_{\mathrm{EW}}}{\sqrt{2} m_{\ell_{i}}} \operatorname{Im}\left(C_{17 Y, i}^{(7)}+\frac{Y_{\chi}}{4} C_{18 Y, i}^{(7)}\right) .
\end{aligned}
$$

The dimension-seven four-fermion UV operators in eq. (3.53)-(3.59) match onto the dimension-seven four-fermion operators in eq. (2.13)-(2.14) after electroweak symmetry breaking. The coefficients for up-type quarks are given by

$$
\begin{aligned}
& \hat{\mathcal{C}}_{19(20), u_{i}}^{(7)}=\frac{1}{2 \Lambda^{3}}\left(-\frac{Y_{\chi}}{4} C_{1(2) F, i}^{(7)}+C_{3(4) F, i}^{(7)}+C_{5(6) F, i}^{(7)}\right), \\
& \hat{\mathcal{C}}_{21(22), u_{i}}^{(7)}=\frac{1}{2 \Lambda^{3}}\left(\frac{Y_{\chi}}{4} C_{1(2) F, i}^{(7)}-C_{3(4) F, i}^{(7)}+C_{5(6) F, i}^{(7)}\right)
\end{aligned}
$$

while for down-type quarks one has

$$
\begin{aligned}
& \hat{\mathcal{C}}_{19(20), d_{i}}^{(7)}=\frac{1}{2 \Lambda^{3}}\left(\frac{Y_{\chi}}{4} C_{1(2) F, i}^{(7)}+C_{3(4) F, i}^{(7)}+C_{7(8) F, i}^{(7)}\right), \\
& \hat{\mathcal{C}}_{21(22), d_{i}}^{(7)}=\frac{1}{2 \Lambda^{3}}\left(-\frac{Y_{\chi}}{4} C_{1(2) F, i}^{(7)}-C_{3(4) F, i}^{(7)}+C_{7(8) F, i}^{(7)}\right) .
\end{aligned}
$$

The matching for charged leptons $\left(\ell_{1}=e, \ell_{2}=\mu, \ell_{3}=\tau\right)$ is given by

$$
\begin{aligned}
& \hat{\mathcal{C}}_{19(20), \ell_{i}}^{(7)}=\frac{1}{2 \Lambda^{3}}\left(\frac{Y_{\chi}}{4} C_{9(10) F, i}^{(7)}+C_{11(12) F, i}^{(7)}+C_{13(14) F, i}^{(7)}\right), \\
& \hat{\mathcal{C}}_{21(22), e_{i}}^{(7)}=\frac{1}{2 \Lambda^{3}}\left(-\frac{Y_{\chi}}{4} C_{9(10) F, i}^{(7)}-C_{11(12) F, i}^{(7)}+C_{13(14) F, i}^{(7)}\right),
\end{aligned}
$$

and for neutrinos $\left(\nu_{1}=\nu_{e}, \nu_{2}=\nu_{\mu}, \nu_{3}=\nu_{\tau}\right)$

$$
\hat{\mathcal{C}}_{19(20), \nu_{i}}^{(7)}=-\hat{\mathcal{C}}_{21(22), \nu_{i}}^{(7)}=\frac{1}{2 \Lambda^{3}}\left(-\frac{Y_{\chi}}{4} C_{9(10) F, i}^{(7)}+C_{11(12) F, i}^{(7)}\right)
$$

Note that the two-Higgs operators in eqs. (3.38)-(3.41) do not contribute to the matching at tree level.

\section{Scalar DM}

Next we turn our attention to complex scalar DM, again allowing for DM to be part of an electroweak multiplet (we comment on the changes required for scalar DM below). There are only two renormalizable couplings between scalar DM and the SM,

$$
\mathcal{L}_{\mathrm{EW}}^{\varphi} \supset \frac{\lambda_{\varphi H}}{4}\left(\varphi^{*} \varphi\right)\left(H^{\dagger} H\right)+\frac{\lambda_{\varphi H}^{\prime}}{4}\left(\varphi^{*} \tilde{\tau}^{a} \varphi\right)\left(H^{\dagger} \tau^{a} H\right) .
$$

If these Higgs-portal couplings are absent or are very small, the higher dimension operators can become important. This can happen, for instance, if $\varphi$ is a pseudo-Goldstone boson in which case the above two operators are forbidden by a shift symmetry. 
The nonrenormalizable interactions of scalar DM with the visible sector start at dimension six,

$$
\mathcal{L}_{\mathrm{EW}}^{\varphi}=\sum_{a, d} \frac{\mathcal{C}_{a}^{(d)}}{\Lambda^{d-4}} Q_{a}^{(d)}(+ \text { h.c. }) .
$$

Note that, in order to simplify the notation, we denote the operators and Wilson coefficients with the same symbols as we did for fermionic DM in the previous two sections. The Hermitian conjugate in the sum is present only when the operators are not Hermitian. This is the case for the operators in eqs. (4.12)-(4.14), whose Wilson coefficients can be complex.

\subsection{Scalar DM above the electroweak scale}

Above the electroweak scale there are 36 Hermitian operators at dimension six that couple scalar DM in a $d_{\chi} \geq 3$ multiplet to the currents in the visible sector. For $d_{\chi}=2$ this reduces to a basis of 33 linearly independent operators, while for $d_{\chi}=1$ the basis is further reduced to only 20 operators (not counting the flavor structures of quark currents). The operators that couple DM currents to the field strength tensors of the SM gauge fields are

$$
\begin{aligned}
Q_{1}^{(6)} & =\frac{\alpha_{s}}{12 \pi}\left(\varphi^{*} \varphi\right) G_{\mu \nu}^{a} G^{a, \mu \nu}, & Q_{2}^{(6)} & =\frac{\alpha_{s}}{8 \pi}\left(\varphi^{*} \varphi\right) G_{\mu \nu}^{a} \tilde{G}^{a, \mu \nu}, \\
Q_{3}^{(6)} & =\frac{\alpha_{1}}{12 \pi}\left(\varphi^{*} \varphi\right) B_{\mu \nu} B^{\mu \nu}, & Q_{4}^{(6)} & =\frac{\alpha_{1}}{8 \pi}\left(\varphi^{*} \varphi\right) B_{\mu \nu} \tilde{B}^{\mu \nu}, \\
Q_{5}^{(6)} & =\frac{\alpha_{12}}{12 \pi}\left(\varphi^{*} \tilde{\tau}^{a} \varphi\right) B_{\mu \nu} W^{a, \mu \nu}, & Q_{6}^{(6)} & =\frac{\alpha_{12}}{8 \pi}\left(\varphi^{*} \tilde{\tau}^{a} \varphi\right) B_{\mu \nu} \tilde{W}^{a, \mu \nu}, \\
\mathcal{Q}_{7}^{(6)} & =\frac{\alpha_{2}}{12 \pi}\left(\varphi^{*} \varphi\right) W_{\mu \nu}^{a} W^{a, \mu \nu}, & Q_{8}^{(6)} & =\frac{\alpha_{2}}{8 \pi}\left(\varphi^{*} \varphi\right) W_{\mu \nu}^{a} \tilde{W}^{a, \mu \nu}, \\
\mathcal{Q}_{9}^{(6)} & =\frac{\alpha_{2}}{12 \pi}\left(\varphi^{*} \tau^{\{a b\}} \varphi\right) W_{\mu \nu}^{a} W^{b, \mu \nu}, & & \mathcal{Q}_{10}^{(6)}=\frac{\alpha_{2}}{8 \pi}\left(\varphi^{*} \tilde{\tau}^{\{a b\}} \varphi\right) W_{\mu \nu}^{a} \tilde{W}^{b, \mu \nu}
\end{aligned}
$$

where we included a loop factor with appropriate gauge couplings, see eq. (3.28). For DM that is an electroweak doublet, $d_{\chi}=2$, the operators $\mathcal{Q}_{9}^{(6)}$ and $\mathcal{Q}_{10}^{(6)}$ should be dropped from the basis as they are not linearly independent, while for DM that is an electroweak singlet, $d_{\chi}=1$, in addition the operators $Q_{5}^{(6)}$ and $Q_{6}^{(6)}$ should be dropped from the basis, since they both vanish in that case.

For $d_{\chi} \geq \mathrm{DM}$ multiplet there are seven operators that involve Higgs currents,

$$
\begin{array}{rlrl}
Q_{11}^{(6)} & =\left(\varphi^{*} \varphi\right)\left(H^{\dagger} H\right)^{2}, & & Q_{12}^{(6)}=\left(\varphi^{*} \tilde{\tau}^{a} \varphi\right)\left(H^{\dagger} \tau^{a} H\right)\left(H^{\dagger} H\right), \\
Q_{13}^{(6)} & =\left(\varphi^{*} \stackrel{\leftrightarrow}{\partial}^{\mu} \varphi\right)\left(H^{\dagger} \stackrel{\leftrightarrow}{\partial}_{\mu} H\right), & & Q_{14}^{(6)}=\left(\varphi^{*} \stackrel{\leftrightarrow}{\partial}^{\mu} \tilde{\tau}^{a} \varphi\right)\left(H^{\dagger} \stackrel{\leftrightarrow}{\partial} \mu \tau^{a} H\right), \\
Q_{15}^{(6)} & =\left(\varphi^{*} i \stackrel{\leftrightarrow}{\partial}^{\mu} \varphi\right) \partial_{\mu}\left(H^{\dagger} H\right), & & Q_{16}^{(6)}=\left(\varphi^{*} i \stackrel{\leftrightarrow}{\partial}^{\mu} \tilde{\tau}^{a} \varphi\right) \partial_{\mu}\left(H^{\dagger} \tau^{a} H\right), \\
\mathcal{Q}_{17}^{(6)} & =\left(\varphi^{*} \tilde{\tau}^{\{a b\}} \varphi\right)\left(H^{\dagger} \tau^{a} H\right)\left(H^{\dagger} \tau^{b} H\right) . &
\end{array}
$$

For DM that is an electroweak doublet, $d_{\chi}=2$, the operator $Q_{17}^{(6)}$ should be dropped from the basis. For singlet DM also the operators $Q_{14}^{(6)}, Q_{16}^{(6)}$ should be dropped. 
The Yukawa-like operators are, for both $d_{\chi} \geq 3$ and $d_{\chi}=2$,

$$
\begin{array}{ll}
Q_{18, i}^{(6)}=\left(\varphi^{*} \varphi\right)\left(\bar{Q}_{L}^{i} u_{R}^{i} \tilde{H}\right), & Q_{19, i}^{(6)}=\left(\varphi^{*} \tilde{\tau}^{a} \varphi\right)\left(\bar{Q}_{L}^{i} u_{R}^{i} \tau^{a} \tilde{H}\right), \\
Q_{20, i}^{(6)}=\left(\varphi^{*} \varphi\right)\left(\bar{Q}_{L}^{i} d_{R}^{i} H\right), & Q_{21, i}^{(6)}=\left(\varphi^{*} \tilde{\tau}^{a} \varphi\right)\left(\bar{Q}_{L}^{i} d_{R}^{i} \tau^{a} H\right), \\
Q_{22, i}^{(6)}=\left(\varphi^{*} \varphi\right)\left(\bar{L}_{L}^{i} \ell_{R}^{i} H\right), & Q_{23, i}^{(6)}=\left(\varphi^{*} \tilde{\tau}^{a} \varphi\right)\left(\bar{L}_{L}^{i} \ell_{R}^{i} \tau^{a} H\right),
\end{array}
$$

For singlet $\mathrm{DM}, d_{\chi}=1$, the operators $Q_{19, i}^{(6)}, Q_{21, i}^{(6)}, Q_{23, i}^{(6)}$ do not appear in the basis. Note that the above operators, $Q_{18, i}^{(6)}, \ldots, Q_{23, i}^{(6)}$ are not Hermitian, and their Wilson coefficients can be complex. Thus, in the effective Lagrangian, eq. (4.2), both the operators $Q_{18, i}^{(6)}, \ldots, Q_{23, i}^{(6)}$ and their Hermitian conjugates appear in the sum. Alternatively, one could use a set of 12 Hermitian operators $\left(6\right.$ for $\left.d_{\chi}=1\right)$, i.e., $\left(Q_{a, i}^{(6)}+Q_{a, i}^{(6) \dagger}\right)$, and $i\left(Q_{a, i}^{(6)}-Q_{a, i}^{(6) \dagger}\right)$, with real Wilson coefficients.

Finally, the operators with fermion currents are, both for $d_{\chi} \geq 3$ and $d_{\chi}=2$,

$$
\begin{aligned}
& Q_{24, i}^{(6)}=\left(\varphi^{*} i \stackrel{\leftrightarrow}{\partial}^{\mu} \varphi\right)\left(\bar{Q}_{L}^{i} \gamma_{\mu} Q_{L}^{i}\right), \quad Q_{25, i}^{(6)}=\left(\varphi^{*} i \stackrel{\leftrightarrow}{\partial}^{\mu} \tilde{\tau}^{a} \varphi\right)\left(\bar{Q}_{L}^{i} \gamma_{\mu} \tau^{a} Q_{L}^{i}\right), \\
& Q_{26, i}^{(6)}=\left(\varphi^{*} i \stackrel{\leftrightarrow}{\partial}^{\mu} \varphi\right)\left(\bar{u}_{R}^{i} \gamma_{\mu} u_{R}^{i}\right), \quad Q_{27, i}^{(6)}=\left(\varphi^{*} i \stackrel{\leftrightarrow}{\partial}^{\mu} \varphi\right)\left(\bar{d}_{R}^{i} \gamma_{\mu} d_{R}^{i}\right), \\
& Q_{28, i}^{(6)}=\left(\varphi^{*} i \stackrel{\leftrightarrow}{\partial}{ }^{\mu} \varphi\right)\left(\bar{L}_{L}^{i} \gamma_{\mu} L_{L}^{i}\right), \quad Q_{29, i}^{(6)}=\left(\varphi^{*} i \stackrel{\leftrightarrow}{\partial}{ }^{\mu} \tilde{\tau}^{a} \varphi\right)\left(\bar{L}_{L}^{i} \gamma_{\mu} \tau^{a} L_{L}^{i}\right), \\
& Q_{30, i}^{(6)}=\left(\varphi^{*} i \stackrel{\leftrightarrow}{\partial} \mu\right)\left(\bar{\ell}_{R}^{i} \gamma_{\mu} \ell_{R}^{i}\right)
\end{aligned}
$$

For singlet DM, $d_{\chi}=1$, the operators $Q_{25, i}^{(6)}$ and $Q_{29, i}^{(6)}$, should be dropped from the basis.

For completeness, we also display the dimension-seven operators for scalar DM. For $d_{\chi} \geq 3$ there are three operators, all of which violate lepton number by two units, $\Delta L=2$,

$$
\begin{aligned}
Q_{1, i}^{(7)} & =\left(\varphi^{*} \varphi\right)\left(\bar{L}_{L, i}^{c} \tilde{H}^{*} \tilde{H}^{\dagger} L_{L, i}\right), \\
Q_{2, i}^{(7)} & =\left(\varphi^{*} \tilde{\tau}^{a} \varphi\right)\left(\bar{L}_{L, i}^{c} \tilde{H}^{*} \tilde{H}^{\dagger} \tau^{a} L_{L, i}\right), \\
Q_{3, i}^{(7)} & =\left(\varphi^{*} \tilde{\tau}^{\{a b\}} \varphi\right)\left(\bar{L}_{L, i}^{c}\left(\tau^{a}\right)^{T} \tilde{H}^{*} \tilde{H}^{\dagger} \tau^{b} L_{L, i}\right) .
\end{aligned}
$$

For DM that is an electroweak doublet, $d_{\chi}=2$, the operator, $Q_{3, i}^{(7)}$ is not linearly independent and should be dropped in order to obtain a basis. For singlet DM both $Q_{2, i}^{(7)}$ and $Q_{3, i}^{(7)}$ are zero.

This completes the construction of the EFT basis for operators with mass dimension seven, for complex scalar DM. We have checked that the basis is complete both with the algorithmic procedure outlined in appendix $\mathrm{A}$, as well as with the conformal Hilbert series method [26-28]. For real scalar DM, the operators $Q_{13}^{(6)}, Q_{15}^{(6)}, Q_{24, i}^{(6)}, Q_{26, i}^{(6)}, Q_{27, i}^{(6)}, Q_{28, i}^{(6)}$, and $Q_{30, i}^{(6)}$ vanish and should be dropped from the basis.

\subsection{Matching below the electroweak scale, RG running, and nuclear response}

Below the electroweak scale the basis for DM interactions with the SM fields contains 8 operators of dimension six (see ref. [25]):

$$
\begin{array}{ll}
\mathcal{Q}_{1, f}^{(6)}=\left(\varphi^{*} i \stackrel{\leftrightarrow}{\partial} \mu\right)\left(\bar{f} \gamma_{\mu} f\right), & \mathcal{Q}_{2, f}^{(6)}=\left(\varphi^{*} i \stackrel{\leftrightarrow}{\partial} \mu\right)\left(\bar{f} \gamma_{\mu} \gamma_{5} f\right), \\
\mathcal{Q}_{3, f}^{(6)}=m_{f}\left(\varphi^{*} \varphi\right)(\bar{f} f), & \mathcal{Q}_{4, f}^{(6)}=m_{f}\left(\varphi^{*} \varphi\right)\left(\bar{f} i \gamma_{5} f\right),
\end{array}
$$




$$
\begin{array}{llrl}
\mathcal{Q}_{5}^{(6)} & =\frac{\alpha_{s}}{12 \pi}\left(\varphi^{*} \varphi\right) G_{\mu \nu}^{a} G_{a}^{\mu \nu}, & \mathcal{Q}_{6}^{(6)} & =\frac{\alpha_{s}}{8 \pi}\left(\varphi^{*} \varphi\right) \tilde{G}_{\mu \nu}^{a} G_{a}^{\mu \nu} \\
\mathcal{Q}_{7}^{(6)} & =\frac{\alpha}{12 \pi}\left(\varphi^{*} \varphi\right) F^{\mu \nu} F_{\mu \nu}, & \mathcal{Q}_{8}^{(6)} & =\frac{\alpha}{8 \pi}\left(\varphi^{*} \varphi\right) F^{\mu \nu} \tilde{F}_{\mu \nu}
\end{array}
$$

Note that the operators $\mathcal{Q}_{1, f}^{(6)}$ and $\mathcal{Q}_{2, f}^{(6)}$ vanish for real DM.

In the remainder of this section we present the explicit tree-level matching conditions from the EFT above the electroweak scale to the five-flavor EFT. The resulting Wilson coefficients can then be evolved, using the RG equations, down to the hadronic scale $\mu \sim 2 \mathrm{GeV}$, where the matching onto the nuclear EFT is performed. The details for the RG running and the subsequent matching to the nuclear EFT for the above operators has been given in ref. [25], with the exception of the Rayleigh operators $\mathcal{Q}_{7}^{(6)}, \mathcal{Q}_{8}^{(6)}$. Their nonperturbative matching is directly analogous to the case of the fermionic operators $Q_{11}^{(7)} \sim$ and $Q_{11}^{(7)}$, for which, however, only NDA estimates are available at present (for the details see the previous section).

Tree-level matching at the electroweak scale gives, for the Wilson coefficients of the operators with the fermionic vector and axial currents in eq. (4.22),

$$
\begin{aligned}
\hat{\mathcal{C}}_{1(2), u_{i}}^{(6)} & =\frac{1}{2 \Lambda^{2}}\left( \pm C_{24, i}^{(6)} \mp \frac{Y_{\chi}}{4} C_{25, i}^{(6)}+C_{26, i}^{(6)}\right), \\
\hat{\mathcal{C}}_{1(2), d_{i}}^{(6)} & =\frac{1}{2 \Lambda^{2}}\left( \pm C_{24, i}^{(6)} \pm \frac{Y_{\chi}}{4} C_{25, i}^{(6)}+C_{27, i}^{(6)}\right), \\
\hat{\mathcal{C}}_{1(2), \ell_{i}}^{(6)} & =\frac{1}{2 \Lambda^{2}}\left( \pm C_{28, i}^{(6)} \pm \frac{Y_{\chi}}{4} C_{29, i}^{(6)}+C_{30, i}^{(6)}\right), \\
\hat{\mathcal{C}}_{1, \nu_{i}}^{(6)} & =-\hat{\mathcal{C}}_{2, \nu_{i}}^{(6)}=\frac{1}{2 \Lambda^{2}}\left(C_{28, i}^{(6)}-\frac{Y_{\chi}}{4} C_{29, i}^{(6)}\right) .
\end{aligned}
$$

The operators involving scalar currents, eq. (4.23), obtain the following contributions:

$$
\begin{aligned}
\hat{\mathcal{C}}_{3, u_{i}}^{(6)}= & \frac{1}{\Lambda^{2}}\left[\frac{v_{\mathrm{EW}}}{\sqrt{2} m_{u_{i}}} \operatorname{Re}\left(C_{18, i}^{(6)}-\frac{Y_{\chi}}{4} C_{19, i}^{(6)}\right)-\frac{v_{\mathrm{EW}}^{2}}{M_{h}^{2}}\left(C_{11}^{(6)}+\frac{Y_{\chi}}{4} C_{12}^{(6)}+\frac{Y_{\chi}^{2}}{8} C_{17}^{(6)}\right)\right] \\
& -\frac{1}{4 M_{h}^{2}}\left(\lambda_{\varphi H}+\frac{Y_{\chi}}{4} \lambda_{\varphi H}^{\prime}\right)+\cdots, \\
\hat{\mathcal{C}}_{4, u_{i}}^{(6)}= & \frac{1}{\Lambda^{2}} \frac{v_{\mathrm{EW}}}{\sqrt{2} m_{u_{i}}} \operatorname{Im}\left(C_{18, i}^{(6)}-\frac{Y_{\chi}}{4} C_{19, i}^{(6)}\right)
\end{aligned}
$$

for up-type quarks,

$$
\begin{aligned}
\hat{\mathcal{C}}_{3, d_{i}}^{(6)}= & \frac{1}{\Lambda^{3}}\left[\frac{v_{\mathrm{EW}}}{\sqrt{2} m_{d_{i}}} \operatorname{Re}\left(C_{20, i}^{(6)}+\frac{Y_{\chi}}{4} C_{21, i}^{(6)}\right)-\frac{v_{\mathrm{EW}}^{2}}{M_{h}^{2}}\left(C_{11}^{(6)}+\frac{Y_{\chi}}{4} C_{12}^{(6)}+\frac{Y_{\chi}^{2}}{8} C_{17}^{(6)}\right)\right] \\
& -\frac{1}{4 M_{h}^{2}}\left(\lambda_{\varphi H}+\frac{Y_{\chi}}{4} \lambda_{\varphi H}^{\prime}\right)+\cdots, \\
\hat{\mathcal{C}}_{4, d_{i}}^{(6)}= & \frac{1}{\Lambda^{3}} \frac{v_{\mathrm{EW}}}{\sqrt{2} m_{d_{i}}} \operatorname{Im}\left(C_{20, i}^{(6)}+\frac{Y_{\chi}}{4} C_{21, i}^{(6)}\right)
\end{aligned}
$$


for down-type quarks, and

$$
\begin{aligned}
\hat{\mathcal{C}}_{3, \ell_{i}}^{(6)}= & \frac{1}{\Lambda^{3}}\left[\frac{v_{\mathrm{EW}}}{\sqrt{2} m_{\ell_{i}}} \operatorname{Re}\left(C_{22, i}^{(6)}+\frac{Y_{\chi}}{4} C_{23, i}^{(6)}\right)-\frac{v_{\mathrm{EW}}^{2}}{M_{h}^{2}}\left(C_{11}^{(6)}+\frac{Y_{\chi}}{4} C_{12}^{(6)}+\frac{Y_{\chi}^{2}}{8} C_{17}^{(6)}\right)\right] \\
& -\frac{1}{4 M_{h}^{2}}\left(\lambda_{\varphi H}+\frac{Y_{\chi}}{4} \lambda_{\varphi H}^{\prime}\right)+\cdots, \\
\hat{\mathcal{C}}_{4, \ell_{i}}^{(6)}= & \frac{1}{\Lambda^{3}} \frac{v_{\mathrm{EW}}}{\sqrt{2} m_{\ell_{i}}} \operatorname{Im}\left(C_{22, i}^{(6)}+\frac{Y_{\chi}}{4} C_{23, i}^{(6)}\right)
\end{aligned}
$$

for charged leptons. The matching for the gluonic operators, eq. (4.24), gives

$$
\hat{\mathcal{C}}_{5(6)}^{(6)}=\frac{1}{\Lambda^{2}} C_{1(2)}^{(6)},
$$

while for the Rayleigh operators we have

$$
\hat{\mathcal{C}}_{7(8)}^{(6)}=\frac{1}{\Lambda^{2}}\left(C_{3(4)}^{(6)}+\frac{Y_{\chi}}{2} C_{5(6)}^{(6)}+C_{7(8)}^{(6)}+\frac{Y_{\chi}^{2}}{2} C_{9(10)}^{(6)}\right) .
$$

Note that, at tree level, the two-Higgs operators with derivatives in eqs. (4.9) and (4.11) do not give contributions to the dimension-six operators below the electroweak scale.

\section{Conclusions}

We presented a complete operator basis for an Effective Field Theory (EFT) that describes the interactions between DM bilinears and the SM fields, up to and including mass dimension seven, both for fermionic and scalar DM. For Dirac fermion and complex scalar DM we restricted the discussion to the case where the EFT obeys a dark global $\mathrm{U}(1)_{D}$ symmetry, under which the DM field carries a nonzero charge. For DM that is a Dirac fermion in a general electroweak $\mathrm{SU}(2)$ multiplet of dimensionality $d_{\chi} \geq 3$, the basis for an EFT valid above the electroweak symmetry breaking scale contains 100 Hermitian dimension-seven operators. We have shown that such a basis is complete. A reduction of the basis occurs, if DM is an electroweak doublet or a singlet, or if DM is a Majorana fermion, as discussed in detail in the main text.

We provided both the tree-level matching at the electroweak scale onto an EFT with five quark flavors, valid for $m_{b} \lesssim \mu \lesssim m_{W}$, as well as the renormalization group running down to an EFT with four quark flavors, valid for $\mu \lesssim m_{b}$, and down to three quark flavor EFT, where also the charm quark is integrated out. We also presented the nonperturbative matching onto the nuclear EFT at leading order in chiral counting.

There are a number of UV models of DM that give the first nonzero contributions only at dimension seven. For instance, if DM is a Majorana fermion, a singlet under the electroweak gauge group, but couples to electroweakly charged heavy states, the leading interaction at low energies can well be the Rayleigh operators, eqs. (2.9) and (2.10) (see ref. [39] for explicit models). Another popular set of UV models that give dimensionseven operators as the leading effect has DM coupling to the visible sector through scalar 
mediators. The scalar mediator exchanges in the $s$-channel (for DM annihilation) lead to the scalar operators in eq. (2.6), while $t$-channel scalar mediator exchanges lead to a linear combination of scalar and tensor operators, eqs. (2.6) and (2.8), after Fierz transformations.

The EFT we presented is valid as long as the mediators are heavier than the momenta exchanges in the physical process one is considering. For direct detection the typical momentum exchange is below $200 \mathrm{MeV}$. This means that the direct detection processes for a large class of the UV theories of DM would be described by the EFT discussed in this paper. Still, one can think of ways to extend the framework further. For instance, one could extend the basis to include operators with several DM multiplets, or to relax the assumption of global dark $\mathrm{U}(1)_{D}$. We leave these for future work.

\section{Acknowledgments}

We thank Fady Bishara for useful discussions. JZ and MT acknowledge support in part by the U.S. National Science Foundation under CAREER Grant PHY-1151392 and by the DOE grant de-sc0011784.

\section{A Construction of the operator basis}

Here we briefly describe the algorithmic procedure that was used to construct the operator basis (see also refs. [35, 40]). We concentrate on the EFT valid above the electroweak breaking scale, so that the DM furnishes a general representation of the $\mathrm{SU}(2)$ gauge group. The construction of the operator basis below the electroweak scale proceeds in an analogous, albeit simpler way.

We start by writing all possible gauge- and Lorentz-invariant contractions of two DM fields with any number of SM fields to construct all possible operators of a given mass dimension. We then use various relations to eliminate redundant operators. Note that we do not include in our basis the operators that involve charge-conjugated dark-matter fields. For Majorana DM these do not lead to any additional operators. However, for Dirac DM one could obtain additional operators, depending on the choice of the DM hypercharge, $Y_{\chi}$, which does not cancel in the $\bar{\chi}^{c} \Gamma \chi$ current (unlike the $\bar{\chi} \Gamma \chi$ currents). For example, for $Y_{\chi}=-1 / 2$ the dimension-seven operator

$$
\left(\bar{\chi}^{c} \chi\right)\left(\bar{L} E^{c} \tilde{H}\right)
$$

is gauge invariant and could be added to the operator basis. Above, the charge conjugation of the DM field $\chi$ includes an appropriate transformation of its $\mathrm{SU}(2)$ representation.

In the remainder of this section we will discuss all relations that were exploited to remove linearly dependent operators. For more details on the actual implementation into C++ code, see ref. [41].

Permutations of identical fields and index symmetries. We explicitly check the disparity of operators under simultaneous permutations of (anti-)symmetric indices and index sets of identical fields, keeping track of fermionic signs. This also allows us to eliminate operators that vanish already via antisymmetry of indices. 
Chirality of standard model fermions. We eliminate all operators that vanish via the chirality of the respective fields.

De-facto symmetry of covariant derivatives. Pairs of covariant derivatives acting on the same field can be decomposed into parts that are symmetric and antisymmetric, respectively. Since the antisymmetric part is proportional to the commutator $\left[D_{\mu}, D_{\nu}\right]$, which is in turn given in terms of gauge field strength tensors, it is already covered by operators with a lower count of covariant derivatives.

Integration by parts. Since total derivatives leave the action invariant, expanding them using the Leibniz rule yields additional relations between our operators.

Fierz identities. The general form of the well-known Fierz identities (see, for instance, refs. $[42,43]$ ) allows us to disregard contractions that are not in a standard order (though this is arbitrarily chosen). Any operator obtained by reordering the pattern of contractions will be a linear combination of the previous operators. This argument also applies to more than two bilinears, since a general permutation of fields can be expressed as a number of transpositions, for which the usual formula holds.

While the Fierz relations can be used to move all bilinears into a standard ordering, there are two cases where they imply additional relations. First, if two fields in the bilinears are identical, application of a Fierz identity directly relates terms that are already in the standard order. Second, if an expression in a non-standard order vanishes, for instance, by chirality, the equation fo motion (EOM), or integration-by-part identities, then the equivalent expression in the standard ordering must vanish too, possibly yielding a new, independent relation.

Schouten identities. These follow from the fact that there is no totally antisymmetric tensor with more indices than the vector space dimension, other than the zero tensor. In our case, antisymmetrizing an object with more than four Lorentz indices yields zero. For instance, antisymmetrizing the indices of the Levi-Civita symbol together with $\mu$ in

$$
\left(\bar{\chi} \sigma_{\mu \nu} \chi\right) B^{\mu}{ }_{\sigma} B_{\alpha \beta} \varepsilon^{\nu \sigma \alpha \beta}
$$

and dropping terms that vanish by symmetry, implies that the whole operator vanishes. Moreover, Schouten identities can also yield relations like

$$
\bar{\chi} \sigma_{\mu \nu} \tau^{a} \chi W_{\sigma}^{b}{ }^{\nu} W_{\alpha \beta}^{c} \varepsilon^{a b c} \varepsilon^{\mu \sigma \alpha \beta}=\bar{\chi} \sigma_{\mu \nu} \tau^{a} \chi W_{\sigma}^{b}{ }^{\alpha} W_{\alpha \beta}^{c} \varepsilon^{a b c} \varepsilon^{\mu \sigma \nu \beta},
$$

which follows from antisymmetrizing the Levi-Civita symbol, together with the index $\nu$, on the left-hand side.

Bianchi identity. The Bianchi identity for any gauge field strength tensor $G_{\mu \nu}^{a}$ is given by

$$
D_{\mu} G_{\nu \rho}^{a}+D_{\nu} G_{\rho \mu}^{a}+D_{\rho} G_{\mu \nu}^{a}=0 .
$$

In particular, this implies that the covariant derivative of the dual tensor vanishes,

$$
D^{\mu} \tilde{G}_{\mu \nu}^{a}=0
$$


Additional reductions for special representations of SU(2). Our operator basis is valid for a general irreducible representation of $\mathrm{SU}(2)$ that the dark matter field $\chi$ furnishes. Additional relations, however, can make some operators redundant for specific representations. For the fundamental representation, $d_{\chi}=2$, some of the operators in the general basis are linearly dependent due to the completeness relation, eq. (3.1). The reduced set of operators is given explicitly in the main text. For the trivial representation of $\mathrm{SU}(2), d_{\chi}=1$, one simply drops all the operators with an $\mathrm{SU}(2)$ generator in the DM current.

Additional reduction for the Majorana/real scalar case. For every Majorana DM bilinear we can use the Majorana condition to replace $\chi$ with its charge conjugate (see, e.g., [44]). A dark matter bilinear that is odd under charge symmetry can then be dropped from the operator basis in the case of Majorana DM, eliminating DM vector and tensor currents (the covariant derivatives acting within the bilinear can change this). The list of these operators is given explicitly at the end of section 3.1. In complete analogy, the operators listed at the end of section 4.1 vanish for real scalar DM.

Equations of motion. The EOM of the SM and DM fields imply relations between operators that can be used to reduce the operator basis [45]. For scalar fields and field strength tensors, the reduction using the EOM is fairly straightforward. For Dirac fields, however, the Clifford algebra complicates matters. To deal with this, we first construct a new set of EOM-vanishing operators by finding all operators of the appropriate mass dimension with any of the following matrix expressions in Dirac field bilinears,

$$
\left\{\gamma^{\mu}, \quad \gamma^{\mu} \gamma_{5}, \quad \gamma^{\mu} \gamma^{\nu}, \quad \gamma^{\mu} \gamma^{\nu} \gamma_{5}, \quad \gamma^{\mu} \gamma^{\nu} \gamma^{\rho}, \quad \gamma^{\mu} \gamma^{\nu} \gamma^{\rho} \gamma_{5}\right\}
$$

where either the rightmost or leftmost Lorentz index is contracted with a covariant derivative acting on the right or left Dirac field, respectively. We then translate each of these terms into relations between elements of our full operator list by exploiting the identities

$$
\begin{aligned}
\gamma_{\mu} \gamma_{\nu} & =\eta_{\mu \nu}-i \sigma_{\mu \nu} \\
\gamma_{\mu} \gamma_{\nu} \gamma_{5} & =\eta_{\mu \nu} \gamma_{5}-i \sigma_{\mu \nu} \gamma_{5} \\
\gamma_{\mu} \gamma_{\nu} \gamma_{\rho} & =\eta_{\mu \nu} \gamma_{\rho}+\eta_{\nu \rho} \gamma_{\mu}-\eta_{\mu \rho} \gamma_{\nu}-i \varepsilon_{\sigma \mu \nu \rho} \gamma^{\sigma} \gamma_{5}, \\
\gamma_{\mu} \gamma_{\nu} \gamma_{\rho} \gamma_{5} & =\eta_{\mu \nu} \gamma_{\rho} \gamma_{5}+\eta_{\nu \rho} \gamma_{\mu} \gamma_{5}-\eta_{\mu \rho} \gamma_{\nu} \gamma_{5}-i \varepsilon_{\sigma \mu \nu \rho} \gamma^{\sigma}
\end{aligned}
$$

where the chirality projector of the SM fields is used to absorb $\gamma_{5}$, if possible. If there already exists another Lorentz Levi-Civita symbol in the remainder of the operator, their product gets simplified as described above.

This procedure, together with the previously discussed treatment of partial integration and index symmetries, captures all relations implied by equations of motion. To see this, consider a completely general EOM-vanishing operator

$$
\left(\bar{\Psi} \Gamma \gamma^{\mu} D_{\mu} \Psi\right) X
$$

where $\Psi$ is any Dirac field, $\Gamma$ is any sum of products of Dirac matrices, possibly contracted with other expressions in the remainder of the operator, which is denoted by $X$. This 
operator, when expressed in our basis, does not induce any relations beyond the ones constructed above. Eqs. (A.9) and (A.10) can be repeatedly applied within each term of $\Gamma$ to reduce it to a sum of terms with a maximum of two gamma matrices (and possibly one $\gamma_{5}$, which can always be anticommuted to the rightmost position and eliminated if an even number of them exists). We have thus expressed the above operator as a linear combination of the vanishing operators already constructed. The case where the covariant derivative acts on the left, or if additional derivatives appear, can be treated analogously.

Finally, as a consistency check of the constructed basis, we compared it with the operator counts for a given field content that were derived using the conformal Hilbert series method [26-28]. This approach allows for a systematic group-theoretic treatment of equations of motion and inclusion of integration-by-parts identities. It calculates an object called the Hilbert series, given by

$$
H\left(\mathcal{D}, \phi_{1}, \ldots, \phi_{N}\right)=\sum_{k, r_{1}, \ldots, r_{N}} c_{k, r_{1}, \ldots, r_{N}} \phi_{1}^{r_{1}} \ldots \phi_{N}^{r_{N}} \mathcal{D}^{k}
$$

where $\phi_{i}$ and $\mathcal{D}$ are complex numbers that stand in for the fields of the theory and the covariant derivative. The coefficients $c_{k, r_{1}, \ldots, r_{N}}$ are the desired operator counts.

The Mathematica package provided in ref. [28], with the addition of the fermionic or scalar DM field in an SU(2) multiplet, gives the total number of independent, Lorentz- and gauge-invariant operators of a certain mass dimension, as well as their field content. We have verified that the number of operators within each operator class obtained in this way exactly matches the number of operators constructed using our algorithmic procedure.

\section{B Non-relativistic reduction of fermion bilinears}

To match onto an EFT with nonrelativistic nucleons, we first introduce a Heavy DM Effective Theory (HDMET) field (for further details see ref. [19]),

$$
\chi(x)=e^{-i m_{\chi} v \cdot x}\left(\chi_{v}(x)+X_{v}(x)\right),
$$

where

$$
\chi_{v}(x)=e^{i m_{\chi} v \cdot x} \frac{1+\psi}{2} \chi(x), \quad X_{v}(x)=e^{i m_{\chi} v \cdot x} \frac{1-\psi}{2} \chi(x) .
$$

Integrating out the antiparticle modes, we obtain the tree-level relation [46]

$$
\chi=e^{-i m_{\chi} v \cdot x}\left(1+\frac{i \not_{\perp}}{i v \cdot \partial+2 m_{\chi}-i \epsilon}\right) \chi_{v}
$$

where $\gamma_{\perp}^{\mu}=\gamma^{\mu}-v^{\mu} \psi$, so that we obtain the conventional "NRQED" type Lagrangian,

$$
\mathcal{L}_{\text {HDMET }}=\chi_{v}^{\dagger}\left(i v \cdot \partial+\frac{\left(i \partial_{\perp}\right)^{2}}{2 m_{\chi}}+\cdots\right) \chi_{v},
$$

where $\partial_{\perp}^{\mu}=\partial^{\mu}-v \cdot \partial v^{\mu}$. Using the replacement (B.3) and applying the equation of motion derived from eq. (B.4), we obtain the following nonrelativistic DM currents:

$$
\begin{aligned}
& \left(\bar{\chi} i \stackrel{\leftrightarrow}{\partial}_{\mu} \chi\right) \rightarrow 2 m_{\chi} v_{\mu} \bar{\chi}_{v} \chi_{v}+\bar{\chi}_{v} i \stackrel{\leftrightarrow}{\partial}_{\mu} \chi_{v}+\ldots, \\
& \left(\bar{\chi} i \gamma_{5} i \stackrel{\leftrightarrow}{\partial}_{\mu} \chi\right) \rightarrow 2 v_{\mu} \partial_{\rho}\left(\bar{\chi}_{v} S_{\chi}^{\rho} \chi_{v}\right)+\frac{1}{m_{\chi}} \partial_{\rho}\left(\bar{\chi}_{v} S_{\chi}^{\rho} i \stackrel{\leftrightarrow}{\partial}_{\mu} \chi_{v}\right)+\ldots
\end{aligned}
$$




$$
\begin{aligned}
\partial_{\mu}\left(\bar{\chi} \sigma^{\mu \nu} \chi\right) \rightarrow & -2 \epsilon^{\alpha \beta \nu \rho} v_{\alpha} \partial_{\beta}\left(\bar{\chi}_{v} S_{\chi, \rho} \chi_{v}\right) \\
& +\frac{v^{\nu}}{2 m_{\chi}}\left(\partial^{2}\left(\bar{\chi}_{v} \chi_{v}\right)-2 \epsilon^{\alpha \beta \rho \sigma} v_{\alpha} \partial_{\rho}\left(\bar{\chi}_{v} S_{\chi, \sigma} i \stackrel{\leftrightarrow}{\partial}_{\beta} \chi_{v}\right)\right)+\ldots, \\
\partial_{\mu}\left(\bar{\chi} \sigma^{\mu \nu} i \gamma_{5} \chi\right) \rightarrow & 2 v^{\nu} \partial_{\rho}\left(\bar{\chi}_{v} S_{\chi}^{\rho} \chi_{v}\right)+\frac{i}{m_{\chi}} \partial_{\rho}\left(\bar{\chi}_{v} S_{\chi}^{\rho} \stackrel{\leftrightarrow}{\partial}^{\nu} \chi_{v}\right)+\cdots,
\end{aligned}
$$

that enter the nonrelativistic reduction of the new dimension-seven operators, eqs. (2.11)(2.14). The ellipses denote higher orders in the expansion in $1 / m_{\chi}$. The remaining nonrelativistic currents, as well as the corresponding products with the expanded nuclear form factors, have already been presented in ref. [19].

Open Access. This article is distributed under the terms of the Creative Commons Attribution License (CC-BY 4.0), which permits any use, distribution and reproduction in any medium, provided the original author(s) and source are credited.

\section{References}

[1] F. Bishara, J. Brod, B. Grinstein and J. Zupan, Chiral effective theory of dark matter direct detection, JCAP 02 (2017) 009 [arXiv:1611.00368] [INSPIRE].

[2] J. Fan, M. Reece and L.-T. Wang, Non-relativistic effective theory of dark matter direct detection, JCAP 11 (2010) 042 [arXiv:1008.1591] [INSPIRE].

[3] A.L. Fitzpatrick et al., The effective field theory of dark matter direct detection, JCAP 02 (2013) 004 [arXiv: 1203.3542] [INSPIRE].

[4] A.L. Fitzpatrick et al., Model independent direct detection analyses, arXiv:1211.2818 [INSPIRE].

[5] N. Anand, A.L. Fitzpatrick and W.C. Haxton, Weakly interacting massive particle-nucleus elastic scattering response, Phys. Rev. C 89 (2014) 065501 [arXiv:1308.6288] [INSPIRE].

[6] M. Cirelli, E. Del Nobile and P. Panci, Tools for model-independent bounds in direct dark matter searches, JCAP 10 (2013) 019 [arXiv:1307.5955] [INSPIRE].

[7] G. Barello, S. Chang and C.A. Newby, A model independent approach to inelastic dark matter scattering, Phys. Rev. D 90 (2014) 094027 [arXiv: 1409.0536] [INSPIRE].

[8] R.J. Hill and M.P. Solon, Standard model anatomy of WIMP dark matter direct detection II: QCD analysis and hadronic matrix elements, Phys. Rev. D 91 (2015) 043505 [arXiv: 1409.8290] [INSPIRE].

[9] R. Catena and P. Gondolo, Global fits of the dark matter-nucleon effective interactions, JCAP 09 (2014) 045 [arXiv: 1405.2637] [INSPIRE].

[10] J. Kopp, T. Schwetz and J. Zupan, Global interpretation of direct dark matter searches after CDMS-II results, JCAP 02 (2010) 014 [arXiv:0912.4264] [INSPIRE].

[11] R.J. Hill and M.P. Solon, WIMP-nucleon scattering with heavy WIMP effective theory, Phys. Rev. Lett. 112 (2014) 211602 [arXiv:1309.4092] [INSPIRE].

[12] R.J. Hill and M.P. Solon, Universal behavior in the scattering of heavy, weakly interacting dark matter on nuclear targets, Phys. Lett. B 707 (2012) 539 [arXiv:1111.0016] [InSPIRE]. 
[13] A. Kurylov and M. Kamionkowski, Generalized analysis of weakly interacting massive particle searches, Phys. Rev. D 69 (2004) 063503 [hep-ph/0307185] [INSPIRE].

[14] M. Pospelov and T. ter Veldhuis, Direct and indirect limits on the electromagnetic form-factors of WIMPs, Phys. Lett. B 480 (2000) 181 [hep-ph/0003010] [INSPIRE].

[15] J. Bagnasco, M. Dine and S.D. Thomas, Detecting technibaryon dark matter, Phys. Lett. B 320 (1994) 99 [hep-ph/9310290] [INSPIRE].

[16] V. Cirigliano, M.L. Graesser and G. Ovanesyan, WIMP-nucleus scattering in chiral effective theory, JHEP 10 (2012) 025 [arXiv: 1205.2695] [INSPIRE].

[17] M. Hoferichter, P. Klos and A. Schwenk, Chiral power counting of one- and two-body currents in direct detection of dark matter, Phys. Lett. B 746 (2015) 410 [arXiv: 1503.04811] [INSPIRE].

[18] M. Hoferichter, P. Klos, J. Menéndez and A. Schwenk, Analysis strategies for general spin-independent WIMP-nucleus scattering, Phys. Rev. D 94 (2016) 063505 [arXiv: 1605.08043] [INSPIRE].

[19] F. Bishara, J. Brod, B. Grinstein and J. Zupan, From quarks to nucleons in dark matter direct detection, JHEP 11 (2017) 059 [arXiv: 1707.06998] [INSPIRE].

[20] J. Menendez, D. Gazit and A. Schwenk, Spin-dependent WIMP scattering off nuclei, Phys. Rev. D 86 (2012) 103511 [arXiv:1208.1094] [InSPIRE].

[21] P. Klos, J. Menéndez, D. Gazit and A. Schwenk, Large-scale nuclear structure calculations for spin-dependent WIMP scattering with chiral effective field theory currents, Phys. Rev. D 88 (2013) 083516 [Erratum ibid. D 89 (2014) 029901] [arXiv: 1304.7684] [INSPIRE].

[22] L. Baudis et al., Signatures of dark matter scattering inelastically off nuclei, Phys. Rev. D 88 (2013) 115014 [arXiv:1309.0825] [INSPIRE].

[23] L. Vietze et al., Nuclear structure aspects of spin-independent WIMP scattering off xenon, Phys. Rev. D 91 (2015) 043520 [arXiv: 1412.6091] [INSPIRE].

[24] J. Goodman et al., Gamma ray line constraints on effective theories of dark matter, Nucl. Phys. B 844 (2011) 55 [arXiv:1009.0008] [inSPIRE].

[25] F. Bishara, J. Brod, B. Grinstein and J. Zupan, DirectDM: a tool for dark matter direct detection, arXiv: 1708.02678 [INSPIRE].

[26] L. Lehman and A. Martin, Hilbert series for constructing lagrangians: expanding the phenomenologist's toolbox, Phys. Rev. D 91 (2015) 105014 [arXiv:1503.07537] [InSPIRE].

[27] B. Henning, X. Lu, T. Melia and H. Murayama, Hilbert series and operator bases with derivatives in effective field theories, Commun. Math. Phys. 347 (2016) 363 [arXiv: 1507.07240] [INSPIRE].

[28] B. Henning, X. Lu, T. Melia and H. Murayama, 2, 84, 30, 993, 560, 15456, 11962, $261485, \ldots$.: higher dimension operators in the SM EFT, JHEP 08 (2017) 016 [arXiv: 1512.03433] [INSPIRE].

[29] J. Brod, B. Grinstein, E. Stamou and J. Zupan, Weak mixing below the weak scale in dark-matter direct detection, JHEP 02 (2018) 174 [arXiv:1801.04240] [INSPIRE].

[30] G. Ovanesyan and L. Vecchi, Direct detection of dark matter polarizability, JHEP 07 (2015) 128 [arXiv:1410.0601] [INSPIRE]. 
[31] T. Appelquist et al., Detecting stealth dark matter directly through electromagnetic polarizability, Phys. Rev. Lett. 115 (2015) 171803 [arXiv:1503.04205] [INSPIRE].

[32] N. Weiner and I. Yavin, How dark are Majorana WIMPs? Signals from MiDM and Rayleigh dark matter, Phys. Rev. D 86 (2012) 075021 [arXiv:1206.2910] [InSPIRE].

[33] M.T. Frandsen et al., Loop-induced dark matter direct detection signals from gamma-ray lines, JCAP 10 (2012) 033 [arXiv: 1207.3971] [INSPIRE].

[34] W. Buchmüller and D. Wyler, Effective Lagrangian analysis of new interactions and flavor conservation, Nucl. Phys. B 268 (1986) 621 [INSPIRE].

[35] B. Grzadkowski, M. Iskrzynski, M. Misiak and J. Rosiek, Dimension-six terms in the standard model lagrangian, JHEP 10 (2010) 085 [arXiv: 1008.4884] [INSPIRE].

[36] F. Bishara, J. Brod, B. Grinstein and J. Zupan, Renormalization group effects in dark matter interactions, arXiv:1809.03506 [INSPIRE].

[37] A. Denner, Techniques for calculation of electroweak radiative corrections at the one loop level and results for $W$ physics at LEP-200, Fortsch. Phys. 41 (1993) 307 [arXiv:0709.1075] [INSPIRE].

[38] M.A. Fedderke, J.-Y. Chen, E.W. Kolb and L.-T. Wang, The fermionic dark matter Higgs portal: an effective field theory approach, JHEP 08 (2014) 122 [arXiv: 1404.2283] [INSPIRE].

[39] N. Weiner and I. Yavin, UV completions of magnetic inelastic and Rayleigh dark matter for the Fermi Line(s), Phys. Rev. D 87 (2013) 023523 [arXiv:1209.1093] [InSPIRE].

[40] B. Gripaios and D. Sutherland, An operator basis for the standard model with an added scalar singlet, JHEP 08 (2016) 103 [arXiv: 1604.07365] [INSPIRE].

[41] A. Gootjes-Dreesbach, Dimension-seven renormalization group effects in an effective field theory approach to fermionic dark matter, M.Sc. thesis, Technischen Universität Dortmund, Dortmund, Germany (2016).

[42] C.C. Nishi, Simple derivation of general Fierz-like identities, Am. J. Phys. 73 (2005) 1160 [hep-ph/0412245] [INSPIRE].

[43] V.I. Borodulin, R.N. Rogalyov and S.R. Slabospitskii, CORE 3.1 (COmpendium of RElations, Version 3.1), arXiv:1702.08246 [INSPIRE].

[44] H.K. Dreiner, H.E. Haber and S.P. Martin, Two-component spinor techniques and Feynman rules for quantum field theory and supersymmetry, Phys. Rept. 494 (2010) 1 [arXiv:0812.1594] [INSPIRE].

[45] H. Simma, Equations of motion for effective Lagrangians and penguins in rare B decays, Z. Phys. C 61 (1994) 67 [hep-ph/9307274] [INSPIRE].

[46] M. Neubert, Heavy quark symmetry, Phys. Rept. 245 (1994) 259 [hep-ph/9306320] [INSPIRE]. 